\title{
Computational Protein Stabilization Can Affect Folding Energy Landscapes and Lead to Domain-Swapped Dimers
}

Klara Markova ${ }^{1,2, \sharp}$, Antonin Kunka ${ }^{1,2, \sharp}$, Klaudia Chmelova ${ }^{1}$, Martin Havlasek $^{1}$, Petra Babkova $^{1}$, Sérgio M. Marques ${ }^{1,2}$, Michal Vasina ${ }^{1}$, Joan Planas-Iglesias ${ }^{1}$, Radka Chaloupkova ${ }^{1}$, David Bednar $^{1,2}$, Zbynek Prokop $^{1,2}$, Jiri Damborsky ${ }^{1,2, *}$, and Martin Marek ${ }^{1,2, *}$

1. Loschmidt Laboratories, Department of Experimental Biology and RECETOX, Faculty of Science, Masaryk University, Kamenice 5, 62500 Brno, Czech Republic

2. International Clinical Research Center, St. Anne's University Hospital Brno, Pekarska 53, 65691 Brno, Czech Republic

$\$$ These authors contributed equally to this study.

*Corresponding authors: jiri@chemi.muni.cz; martin.marek@ recetox.muni.cz 


\begin{abstract}
The functionality of a protein depends on its unique three-dimensional structure, which is a result of the folding process when the nascent polypeptide follows a funnel-like energy landscape to reach a global energy minimum. Computer-encoded algorithms are increasingly employed to stabilize native proteins for use in research and biotechnology applications. Here, we reveal a unique example where the computational stabilization of a monomeric $\alpha / \beta$ hydrolase enzyme $\left(T_{\mathrm{m}}=73.5^{\circ} \mathrm{C} ; \Delta T_{\mathrm{m}}>23^{\circ} \mathrm{C}\right)$ affected the protein folding energy landscape. Introduction of eleven single-point stabilizing mutations based on force field calculations and evolutionary analysis yielded catalytically active domain-swapped intermediates trapped in local energy minima. Crystallographic structures revealed that these stabilizing mutations target cryptic hinge regions and newly introduced secondary interfaces, where they make extensive non-covalent interactions between the intertwined misfolded protomers. The existence of domain-swapped dimers in a solution is further confirmed experimentally by data obtained from SAXS and crosslinking mass spectrometry. Unfolding experiments showed that the domain-swapped dimers can be irreversibly converted into native-like monomers, suggesting that the domain-swapping occurs exclusively in vivo. Our findings uncovered hidden protein-folding consequences of computational protein design, which need to be taken into account when applying a rational stabilization to proteins of biological and pharmaceutical interest.
\end{abstract}

\title{
KEYWORDS
}

protein folding; protein design; $\alpha / \beta$-hydrolase, haloalkane dehalogenase; domain-swapping; dimerization; energy landscape; oligomerization 


\section{INTRODUCTION}

Protein folding is a process in which a polypeptide chain folds into its native state, a welldefined three-dimensional structure, which is a prerequisite for its proper biological function. During this process, a polypeptide chain undergoes many conformational changes as it navigates through the partially folded states down the energy landscape to the free energy minimum - a native state. ${ }^{1}$ The folding energy landscape of a protein is encoded by its amino acid sequence. Consequently, any modification of the protein's sequence may alter its folding pathway. Energy landscapes of natural proteins are shaped by the evolution towards functional structures. Protein engineering aims to modulate the protein sequence so that the native state is able to perform its function even under unnatural conditions. Computationally designed substitutions are typically introduced to create favorable stabilizing interactions in the native state. ${ }^{2-4}$ Despite numerous, and often remarkably successful, attempts at stabilization $^{3,5,6}$, the associated changes to the folding energy landscape have not been investigated, to the best of our knowledge.

Domain swapping ${ }^{7,8}$ is a specific form of protein oligomerization, in which small elements or domains are exchanged among identical polypeptide chains. ${ }^{9,10}$ Domain swapping can result in dimers, closed oligomers or filament-like oligomers. ${ }^{8}$ The mechanism of domain swapping requires either nearly complete unfolding ${ }^{11}$ or opening of the protein into a partially unfolded state, allowing the exchange of the structural elements between two or more identical chains. ${ }^{12}$ Most interactions that are crucial for stabilizing a monomeric form are also preserved in the domain-swapped structure. Additionally, new interactions can be formed in the domain-swapped structures and create a secondary interface. ${ }^{13}$ A hinge region is a protein segment that adopts different backbone conformations in the monomer and domain-swapped oligomer, connecting the swapped and the non-swapped domains of the single polypeptide chain. ${ }^{14}$ The hinge region acts as a conformational switch, with its length and sequence composition being critical for the domain swapping propensity. ${ }^{10}$ Engineering of putative hinge loops is an attractive strategy for de novo domain-swapping design. ${ }^{15,16}$

Domain swapping can be biologically relevant as a regulatory mechanism, for example, in receptor binding proteins ${ }^{17}$ or DNA-binding proteins. ${ }^{18,19}$ It has been reported as an effective strategy for protein function modification in vitro ${ }^{20}$ and an analogous mechanism was utilized in the successful design of the ligand-triggered functional switch of staphylococcal nuclease and ribose binding protein. ${ }^{21}$ Domain swapping was also proposed as one of the evolutionary 
mechanisms of protein oligomerization. ${ }^{22}$ A possible link between domain swapping and protein deposition diseases has been implied. ${ }^{23}$ Crystal structures of human prion protein and cystatin $\mathrm{C}$, involved in the prion and amyloid diseases, respectively, revealed the formation of dimers through a domain-swapping mechanism. ${ }^{24,25}$ Structural and functional studies of domain swapping are essential for our understanding of this phenomenon.

In this study, we show that the computationally-driven stabilization of a monomeric haloalkane dehalogenase DhaA115 $\left(T_{\mathrm{m}}{ }^{\text {app }}=73.5^{\circ} \mathrm{C} ; \Delta T_{\mathrm{m}}{ }^{\text {app }}>23^{\circ} \mathrm{C}\right)^{5,26}$ unintentionally altered the protein folding landscape, resulting in the formation of stable domain-swapped intermediates. Our structural findings reveal that the intended stabilizing mutations were frequently found in the cryptic hinge regions and introduced secondary interfaces where they made new non-covalent interactions between the misfolded, intertwined polypeptide chains. We further demonstrate that the domain-swapped dimers can be irreversibly converted into native-like monomers using thermal or chemical denaturation, suggesting that the domain swapping occurs exclusively in vivo. Collectively, our results highlight an unprecedented example of when computationally guided protein stabilization is unexpectedly accompanied by the in-cell formation of misfolded domain-swapped intermediates.

\section{RESULTS}

De Novo Oligomerization Due to Computer-Aided Stabilization. We have already reported that our previous computer-aided engineering of a haloalkane dehalogenase DhaA from Rhodococcus rhodochrous was unexpectedly accompanied by de novo formation of enzyme oligomers. ${ }^{26,27}$ While the DhaA wild-type enzyme $\left(T_{\mathrm{m}}{ }^{\text {app }}=\sim 50.4^{\circ} \mathrm{C}\right)$ exists solely in a monomeric form, the most stabilized 11-point mutant DhaA115 $\left(T_{\mathrm{m}}{ }^{\text {app }}=\sim 73.4^{\circ} \mathrm{C}\right)$ exists in monomeric $(\sim 75.7 \%)$, dimeric $(\sim 20.3 \%)$ and higher oligomeric $(\sim 4.0 \%)$ forms (Figure 1 and Table S1). A single band of $\sim 35 \mathrm{kDa}$ can be detected using electrophoresis in denaturing conditions for both wild-type DhaA and DhaA115, while native non-denaturing electrophoretic analysis revealed multiple distinct oligomeric states of hyperstable DhaA115 (Figure 1A). Notably, the gel-separated fractions corresponding to the putative dimeric form of DhaA115 repeatedly migrated as a specific double band, suggesting the existence of different conformers. Analytical size exclusion chromatography (SEC) and analytical ultracentrifugation (AUC) of DhaA115 confirmed the presence of a monomeric, dimeric and a 
small amount of non-specified oligomers of higher molecular weights (Figures 1B-C). Further SEC analysis at increased protein concentrations showed that the oligomeric content was independent of protein concentration (Table S1).

A
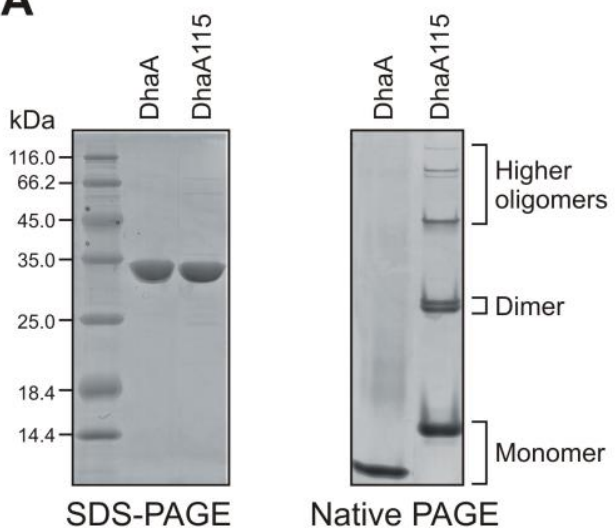

D

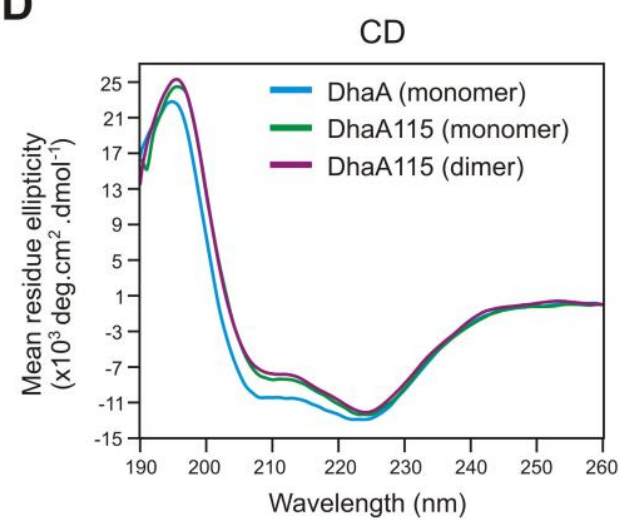

B

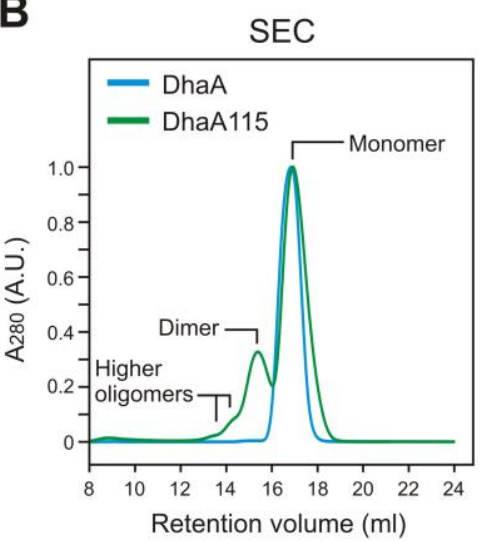

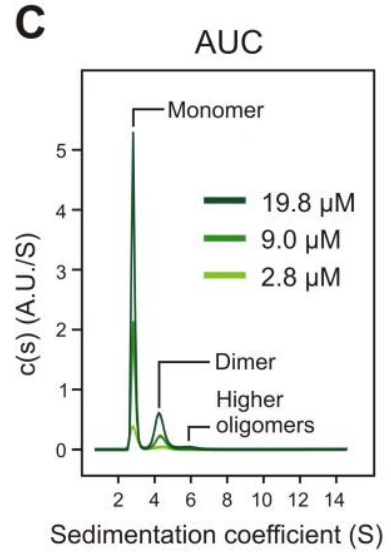

E

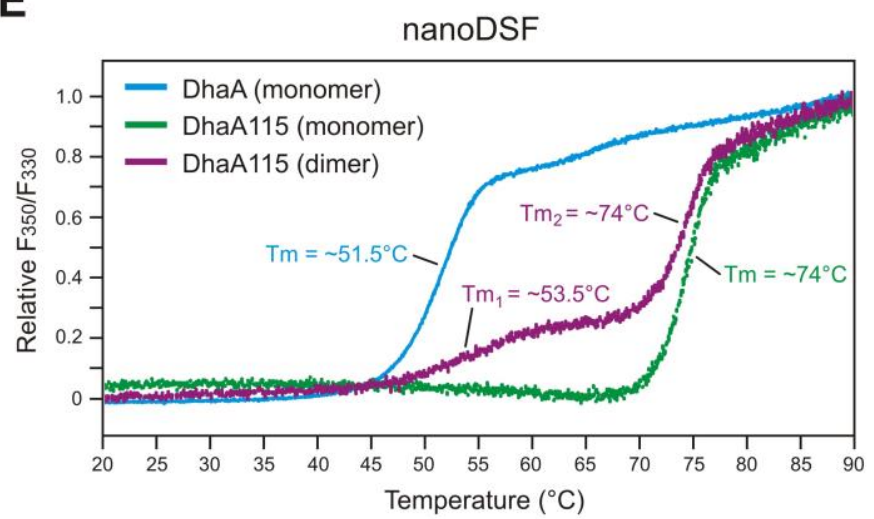

Figure 1. Biophysical and biochemical characterization of the hyperstable engineered DhaA115. (A) Electrophoretic separation of DhaA and DhaA115 proteins by SDS-PAGE (left panel) and native nondenaturing PAGE (right panel). (B) Analysis of DhaA and DhaA115 by analytical size-exclusion chromatography (SEC). (C) Analysis of DhaA115 by analytical ultracentrifugation (AUC). (D) Circular dichroism spectra of DhaA monomers, DhaA115 monomers and DhaA115 dimers. (E) Differential scanning fluorimetry (nanoDSF) of DhaA monomers, DhaA115 monomers and DhaA115 dimers. Note that the melting of DhaA115 dimers is accompanied by two major melting points: $T_{\mathrm{m} 1}=$ $53.5^{\circ} \mathrm{C}$ and $T_{\mathrm{m} 2}=74^{\circ} \mathrm{C}$.

Next, we carried out circular dichroism (CD) spectroscopy on SEC-purified protein fractions to check the effect of DhaA115 oligomerization on protein folding. The shapes of the CD spectra of all measured species showed characteristics typical of an $\alpha / \beta$-hydrolase fold, specifically one positive peak at 195-197 nm and two negative minima at approximately 
$209 \mathrm{~nm}$ and $224 \mathrm{~nm}$. CD spectra of DhaA115 monomeric and dimeric forms were highly similar, indicating that the secondary structure of DhaA115 monomer was preserved in its dimeric form (Figure 1D). Finally, differential scanning fluorimetry experiments identified similar melting transitions with the midpoint at $74{ }^{\circ} \mathrm{C}$, preceded by an additional minor transition with midpoint around $53.5^{\circ} \mathrm{C}$ observed in the case of the DhaA115 dimer (Figure $1 \mathrm{E})$.

DhaA115 Oligomerizes Through Domain-Swapping Mechanism. To gain structural insights into the mechanism of DhaA115 oligomerization, we attempted crystallization of its oligomeric forms. Despite intensive efforts, we were not able to obtain well-diffracting crystals of the Dha1 15 higher oligomeric species. In contrast, the crystallization of the DhaA115 dimeric form was successful and yielded two types of well-diffracting crystals. The first belonged to the space group $P 2{ }_{1} 2{ }_{1}{ }_{1}$, whilst the second crystal form belonged to the $P 12{ }_{1} 1$ space group (Table S2). The structures were solved by molecular replacement, and the initial models were further refined with several cycles of manual building and automatic refinement, yielding structural models with good deviations from ideal geometry (Table S2). Most of the residues could be built in electron density, except for a few residues on Nterminal and C-terminal ends.

Surprisingly, careful inspection of the electron density maps unambiguously revealed two intertwined polypeptide chains (protomers), which formed dimers through a domainswapping mechanism (Figure 2). Importantly, the DhaA115 domain-swapped dimer structure solved in the space group $P 2{ }_{1} 2{ }_{1}{ }_{1}$, hereafter referred to as DhaA115-DSD1, structurally differed from that found in the second type of crystals processed in space group $P 12{ }_{1} 1$, which is further referred to as DhaA115-DSD2. Both DhaA115 domain-swapped dimers differ from each other in the position of the hinge loop, the only region of the protein that adopts a different conformation in the monomeric and domain-swapped dimeric structures. 
A

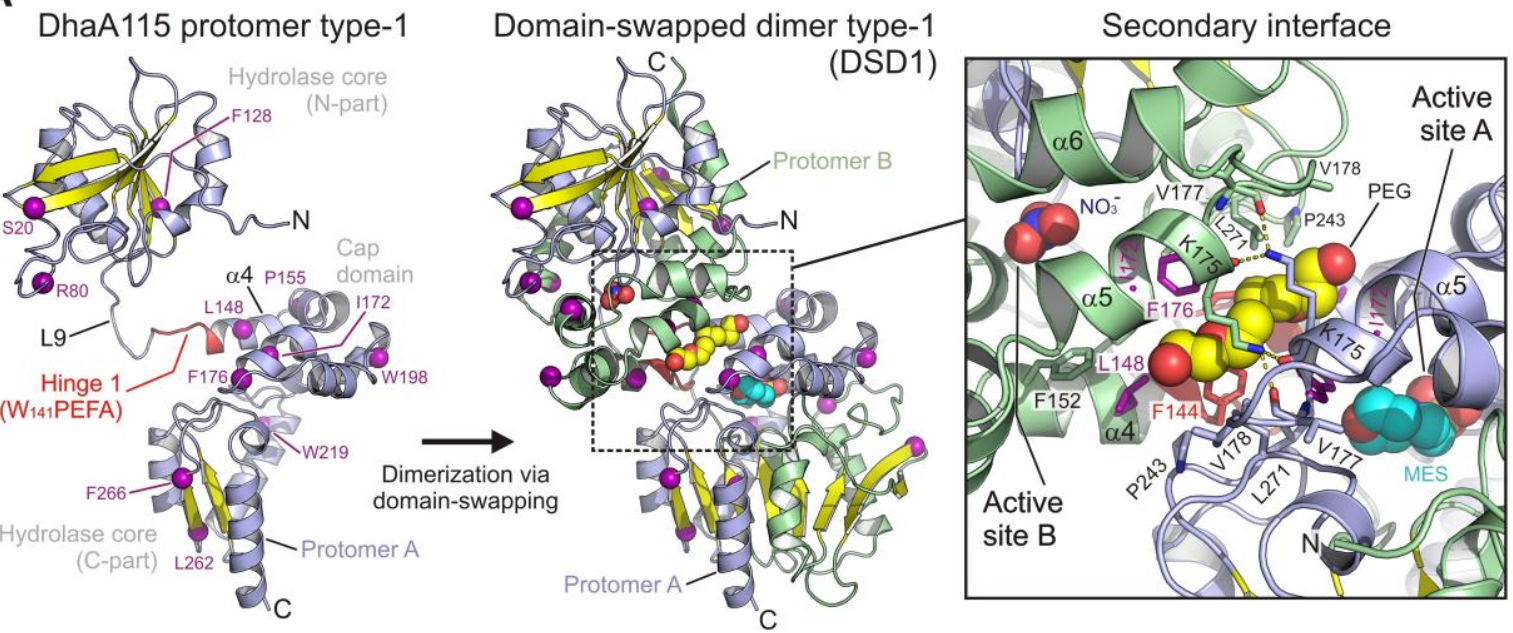

B
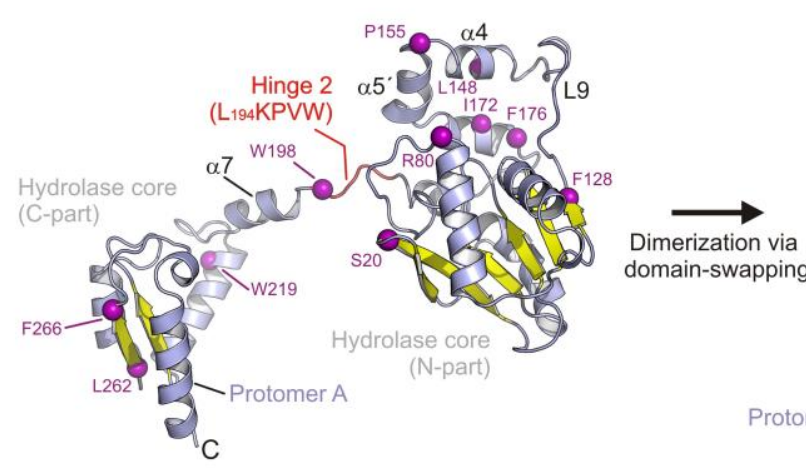

Hinge 2 region and secondary interface
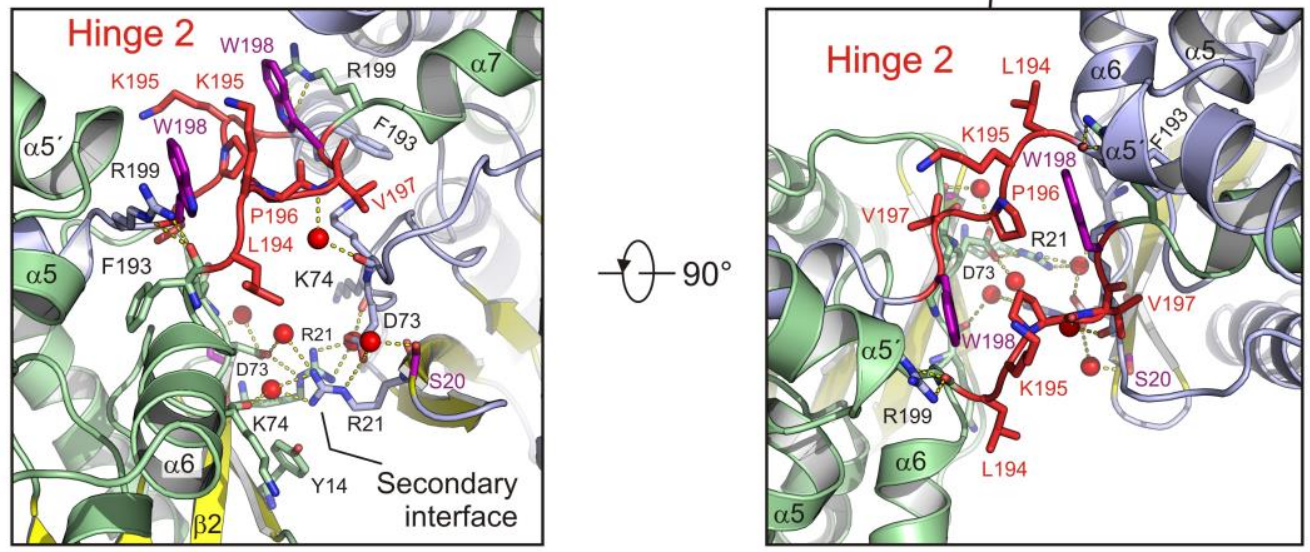

Figure 2. X-ray structures of domain-swapped dimers DhaA115-DSD1 and DhaA115-DSD2. (A) Cartoon representations of DhaA115 protomer type-1 (left panel), DhaA115-DSD1 dimer (middle panel), and close-up view of the new secondary interface (right panel). The central eight-stranded $\beta$ sheet (yellow), the helices (light blue) and the hinge region $1\left(\mathrm{~W}_{141} \mathrm{PEFA}\right)$ sequence (red). The designed stabilizing mutations are shown as purple spheres; the polyethylene glycol (PEG) molecule bound between the two catalytic units is shown as yellow spheres; the 2-(Nmorpholino)ethanesulfonic acid (MES) bound in the active site A is shown as cyan spheres; and the 
nitrate $\left(\mathrm{NO}^{3-}\right)$ anion bound in the active site $\mathrm{B}$ is shown as blue spheres. The key residues involved in the secondary interface are shown as sticks; hydrogen bonds are shown as yellow dashed lines. (B) Cartoon representations of DhaA115 protomer type-2 (left panel), DhaA115-DSD2 dimer (right panel), and close-up views of the hinge 2 region and new secondary interface (bottom panels). Colorcoding is same as in A panels. The hinge 2 region $\left(\mathrm{K}_{194} \mathrm{PVW}\right)$ sequence (red); and the chloride $\left(\mathrm{Cl}^{-}\right)$ anions bound in the active sites are shown as green spheres.

The dimeric structure of DhaA115-DSD1 is mediated by the altered conformation of L9 loop, which connects the $\beta 6$ strand with the cap domain-forming $\alpha 4$ helix (Figure 2A). Specifically, the $\mathrm{W}_{141}$ PEFA element of L9 loop functions as the hinge region in DhaA115DSD1. As a consequence, the $\alpha 4$ helix is slightly deflected (by an angle of $\sim 10^{\circ}$ ) when compared to its monomeric counterpart. The structure of DhaA115-DSD1 further reveals a new secondary interface, a site where the two intertwined protomers extensively interact with each other and thus stabilize the domain-swapped dimer (Figure 2A). The secondary interface has a predominantly hydrophobic and aromatic character (F144, L148, I172, and F176), although polar contacts and hydrogen bonding are also involved (K175, V177, P249, and A250). The secondary interface shapes a pore with a diameter of $\sim 4-5 \AA$ between the intertwined protomers, where an unambiguous linear electron density occupying this pore is present (Figure 2A). We interpreted this electron density as a polyethylene glycol molecule which was one of the components of the crystallization buffer. Interestingly, three residues introduced into DhaA115 by the computational protein design as stabilizing mutations (L148, I172 and F176) ${ }^{5}$ are involved in the formation of this secondary interface.

A structurally distinct domain-swapping topology was observed in the crystal structure of DhaA115-DSD2, where the asymmetric unit consisted of two domain-swapped dimers. Domain swapping in DhaA115-DSD2 is mediated by the conformational change of L13 loop (residues $\mathrm{L}_{194} \mathrm{KPVW}$ ). The L13 loop connects $\alpha 6$ and $\alpha 7$ helices, which are part of the cap domain (Figure 2B). Interestingly, the DhaA115-DSD2 dimer does not contain an extensive stabilizing secondary interface as observed in the case of DhaA115-DSD1. At the DhaA115DSD2 secondary interface, two opposite arginine residues, R21, from the individual protomers form an arginine pair, whose repulsive nature is stabilized by hydrogen bonding with the negatively charged D73 and bound water molecules (Figure 3B). The serine residue S20 designed by computational stabilization ${ }^{5}$ is present in the vicinity of the DhaA115-DSD2 secondary interface, although it does not seem to be directly participating in its formation. 
The other molecular interactions between the two protomers in DhaA115-DSD2 are facilitated by the hinge region, which allows intrinsic conformational flexibility of the dimeric structure. This conformational freedom is apparent from the superimposition of the two noncrystallographic DhaA115-DSD2 dimers found in the asymmetric unit (Figure S1). Importantly, the hinge region in DhaA115-DSD2 is composed of the sequence element $\mathrm{L}_{194} \mathrm{KPVW}$, where the pyrrolidine rings of the two opposing prolines, P196, from each protomer are arranged around the non-crystallographic two-fold axis (Figure 2B). These hinge regions interact with each other through multiple non-polar and hydrophobic contacts, thus forming the two-loop bundle that bridges the two pseudo- $\alpha / \beta$-hydrolase folds. Importantly, one of the stabilizing mutations, D198W, is located in the DhaA115-DSD2 hinge region. ${ }^{5}$

DhaA115 Domain-Swapped Dimers Exist in Solution. We speculated as to whether the domain-swapped DhaA115 dimers could be a crystallographic artefact. We initially probed SEC-separated dimeric fractions of the DhaA115 enzyme using a small-angle X-ray scattering (SAXS) technique. The SAXS profile (Figure 3A) fits well to the scattering profile calculated using both crystallographic dimers, although the fit for DhaA115-DSD2 structure $\left(\chi^{2}=2.40\right)$ is slightly better than that for DhaA115-DSD1 $\left(\chi^{2}=4.62\right)$. The radius of gyration $\left(R_{\mathrm{g}}\right)$ obtained from the SAXS data was $\sim 29.5 \AA$. The profile of the pair distance distribution function has a double bell-like shape with the main peak at $\sim 27.2 \AA$, and trails off to the maximum dimension $\left(D_{\max }\right)$ of $\sim 89.2 \AA$. The ab initio model reconstructed from the SAXS data perfectly matches the DhaA115-DSD2 dimer, while there are observable discrepancies between the SAXS envelope and the DhaA115-DSD1 crystal structure (Figure 3A). We also calculated the $R \mathrm{~g}$ values for the molecular dynamics (MD) simulations: $R_{\mathrm{g}}=17.83 \pm 0.04 \AA$ for the monomeric DhaA115, $R_{\mathrm{g}}=26.89 \pm 0.11 \AA$ for DhaA115-DSD1, and $R_{\mathrm{g}}=28.61 \pm 0.16 \AA$ for DhaA115-DSD2. Notably, the latter value for DhaA115-DSD2 is very close to the SAXSdetermined value for this dimer $(\sim 29.5 \AA)$. 
A
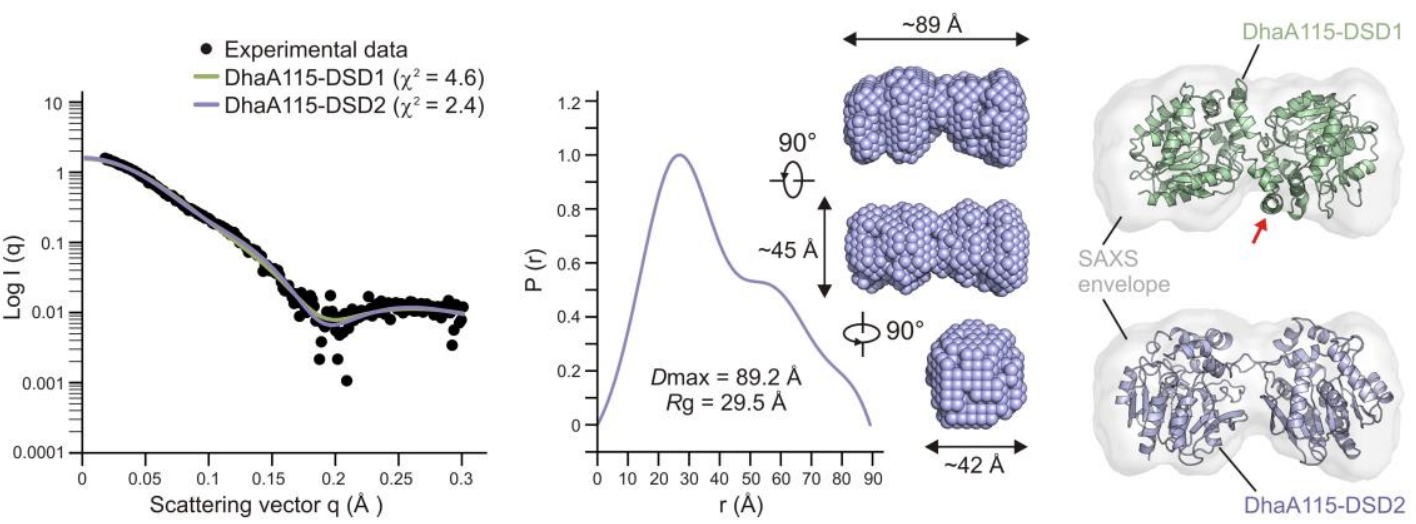

B

C4-urea-NHS-crosslinker

(DSBU)<smiles>O=C(NCCCC(=O)ON1C(=O)CCC1=O)NCCCC(=O)ON1C(=O)CCC1=O</smiles>

C

DhaA115 monomer

DhaA115 dimer
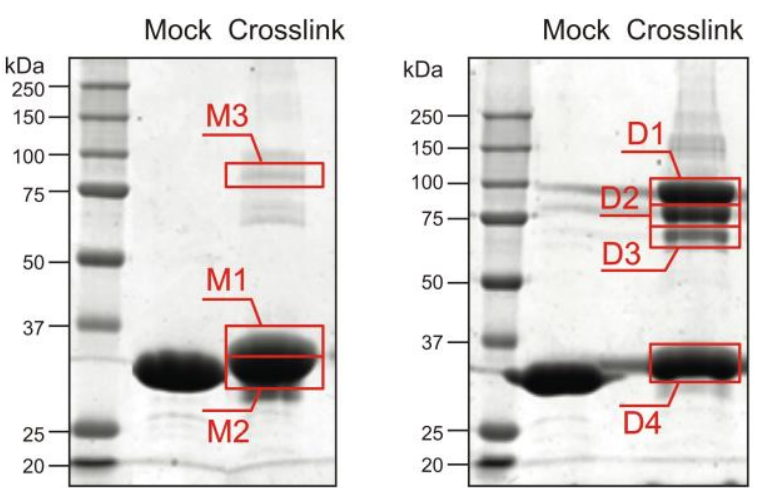

E

DhaA115-DSD2
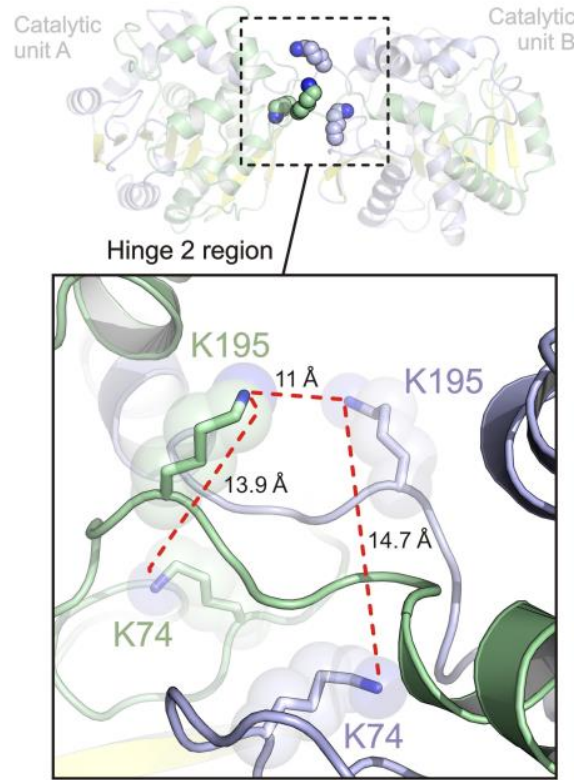

\begin{tabular}{cccccccc}
\hline Dample & $\begin{array}{c}\text { Merox } \\
\text { score }\end{array}$ & Site 1 & Peptide 1 & Site 2 & Peptide 2 & $\begin{array}{c}\text { Probability } \\
(\%)\end{array}$ & $\begin{array}{c}\text { No. matching } \\
\text { spectra }\end{array}$ \\
\hline M1 & - & - & - & - & - & - & - \\
\hline M2 & 171 & 195 & EPFLKPVWR & 74 & SDKPDLDYR & 80 & 4 \\
\hline M3 & - & - & - & - & - & - & - \\
\hline D1 & 218 & 195 & EPFLKPVWR & 195 & EPFLKPVWR & 100 & 7 \\
\hline D2 & 206 & 195 & EPFLKPVWR & 195 & EPFLKPVWR & 100 & 7 \\
& 187 & 195 & EPFLKPVWR & 74 & $\begin{array}{c}\text { SDKPDLDYR/BIAPDLI } \\
\text { GMGKSDKPDLDYR }\end{array}$ & 76 & 13 \\
\hline D3 & 207 & 195 & EPFLKPVWR & 195 & EPFLKPVWR & 100 & 3 \\
& 115 & 195 & EPFLKPVWR & 74 & SDKPDLDYR & 77 & 2 \\
\hline D4 & - & - & - & - & - & - & - \\
\hline
\end{tabular}

Figure 3. Characterization of the DhaA115 dimers by small-angle X-ray scattering (SAXS) and crosslinking coupled mass spectrometry (XL-MS). (A) Solution structure of DhaA115 dimers determined by SAXS. Experimental SAXS scattering curve for DhaA115 dimer (black dots) is shown against the calculated scattering curves derived from the DhaA115-DSD1 (green line) and DhaA115-DSD2 (blue 
line) crystal structures (left panel). The distance distribution function of DhaA115 dimer computed from the X-ray scattering pattern using the GNOM program (middle panel). Ab initio molecular envelope generated from SAXS data shown in semi-transparent grey colour and superposed on the DhaA115-DSD1 (green) and DhaA115-DSD2 (blue) crystal structures (right panels). (B) The structural formula of C4-urea-NHS-crosslinker (DSBU). (C) SDS-PAGE separation of crosslinkertreated DhaA115 monomers (left panel) and DhaA115 dimers (right panel). Red frames (M1-3 and D1-4) depict protein specimens that were excised, processed and analyzed by LC-MS/MS. (D) Table of crosslinked peptides identified in separated fractions (M1-3 and D1-4) by LC-MS/MS analysis. MeroX software detected homo-dipeptides (EPFLK ${ }_{195}$ PVWR) only in the samples D1 and D2 that originated from DhaA115 dimers. The lysine residues that were covalently crosslinked are in bold. (E) Cartoon representation of DhaA115-DSD2 structure with shown lysine residues (K74 and K195) present in the hinge region and its vicinity. Lysine-to-lysine covalent links mediated by DSBU reagent are shown as red dashed lines.

We further employed chemical crosslinking coupled with mass spectrometry (XL-MS) to capture domain-swapped dimers in a solution. The crosslinking experiment for the SECpurified DhaA115 monomers with a cleavable 11-atom crosslinker (4,4'-ureylenedibutyric acid bis-NHS ester) produced complexes that contained intramolecular hetero-dipeptides crosslinked at lysines K74 and K195 (Figure 3B-D). In contrast, two major complexes were detected in the same experiment for the dimeric fraction of DhaA115. The first one was the same intramolecular hetero-dipeptide between lysine residues K74 and K195 as observed in the DhaA115 monomeric fraction (Figure 3B-D). The second covalently crosslinked complex, exclusively detected in the dimeric fraction of DhaA115, contained intermolecular homodipeptides formed between lysines K195 of the two individual polypeptide chains. The distance between lysines K74 and K195 in the crystal structures of both monomeric and dimeric forms of DhaA115 was 12-14 $\AA$, which corresponds to the length of the crosslinker $(12.5 \AA)$ used in the experiment. This explains why the intramolecular hetero-dipeptide created between these lysines was detected in both monomeric and dimeric fractions when crosslinked.

By contrast, the intermolecular homo-dipeptides linked through lysine K195 were detected solely in the dimeric fraction (Figure 3B-D). Careful inspection of the crystal structures revealed that the crosslinked homo-dipeptides detected most likely originated from the DhaA115-DSD2, since lysine K195 is located in the hinge region of this conformer. As a 
result, lysines K195 from the individual protomers move close to this region, and are $\sim 11 \AA$ apart (Figure 3E). In contrast, the distance between these identical residues in the two protomers of DhaA115-DSD1 is $55 \AA$, too far for a successful crosslinking with the 11-atom crosslinker. Collectively, the results of SAXS and XL-MS experiments prove that DhaA115 domain-swapped dimers exist in solution and imply that the DhaA115-DSD2 is the dominant species.

MD Simulations Imply Higher Flexibility of the Domain-Swapped Dimers. Next, we used MD simulations to study the conformational dynamics of the domain-swapped dimers in solution. Multiple MD simulations of $100 \mathrm{~ns}$ length were carried out with the two dimeric structures, and their flexibility analyzed using root-mean-square deviation (RMSD) of the atomic positions. These simulations were reasonably stable after $100 \mathrm{~ns}$, as assessed by the plateaus observed in the respective RMSD plots near the end of each MD (Figure S2). Interestingly, the two domain-swapped dimers deviated from their respective crystal structures considerably with RMSD fluctuations above $2-3 \AA$, in contrast to the relatively rigid structures of monomeric DhaA115 and wild-type DhaA (RMSD below $1 \AA$ ). ${ }^{26}$

To investigate the main reason for such large deviations, we clustered those trajectories by the RMSD and analyzed the centroid structures (Figures S3 and S4). We found that the structural changes consisted mainly of the repositioning of the two globular units with respect to each other, while each catalytic unit remained very stable and superimposable with itself (Figures S3C and S4C). The large topological changes observed during the MD simulations are caused by the conformational freedom of the hinge regions of the domain-swapped dimers. Domain-swapped dimers of DhaA115 can adopt spatially different conformations in the crystal state and in solution, which is most likely influenced by (i) the length and flexibility of the hinge region, (ii) nature of the secondary interface, and possibly (iii) buffer composition. For instance, in the MD simulations of DhaA115-DSD1, the most populated state corresponds to the cluster with the largest deviation (cluster 1, RMSD $2.75 \pm 0.20 \AA$ ), which was also the dominant conformation by the end of both MD simulations. Conversely, the most populated conformation during MD simulation of DhaA115-DSD2 was the one closest to the crystal structure (cluster 1 , RMSD $1.24 \pm 0.20 \AA$ ), and the most deviated one was populated the least (cluster 4, RMSD $1.82 \pm 0.22 \AA$ ).

The B-factors of the protein backbone were calculated as a measure of the respective residue flexibility. The B-factors of the two dimers are higher than for the monomer and 
greater for DhaA115-DSD1 than for DhaA115-DSD2 (Figure S5A). This is in agreement with the RMSD results described above. However, we noted that most of these fluctuations were due to the rocking and tilting movements of the two catalytic units with respect to one another. Therefore, we carried out a stepwise analysis of the MDs by aligning the systems to each catalytic unit at the time, calculating the respective B-factors, and then combining them. These new results (Figures S5B and S6) showed that DhaA115-DSD1 is still the most flexible of the DhaA115 variants tested here. DhaA115-DSD2 showed similar B-factors to those observed for the monomeric DhaA115 when considering the individual catalytic units, and even slightly lower for some residues.

\section{Domain-Swapped Dimer Opens and Collapses to Monomers During Thermal}

Unfolding. We used protein unfolding analysis to resolve the mechanism by which the domain-swapped dimers of DhaA115 form. The unfolding of wild-type monomeric DhaA proceeds through one or more intermediates (unpublished data). Refolding of DhaA from a heat-denatured state leads to a mixture of a native-like state and an ensemble of aggregated states of various sizes. We hypothesized that the domain-swapping of DhaA115 proceeds with similar intermediate formation during refolding, while the introduced mutations stabilize local minima on the protein folding landscape. To test this hypothesis, we carried out unfolding and refolding experiments with monomeric and dimeric fractions of DhaA115 using circular dichroism (CD) and fluorescence spectroscopy in combination with differential scanning calorimetry (DSC). The monomer unfolding shows a single transition with the midpoint at $\sim 73^{\circ} \mathrm{C}$ when monitored with spectroscopic techniques (Figure S7) and can be fitted together globally with calorimetric and unfolding kinetics data to the three-state partially reversible mechanism (Lumry-Eyring model) ${ }^{28,29}$. Refolding was carried out by heating the monomer at various concentrations to different temperatures, to and above the unfolding transition range, and cooling it down at the same rate $\left(1^{\circ} \mathrm{C} / \mathrm{min}\right)$. The resulting oligomeric states of the sample were then analyzed using SEC and native PAGE. As seen with the wild-type, refolding of DhaA115 yielded a mixture of monomeric protein and aggregates (Figure S8A). The fraction of the aggregated states was concentration-dependent and increased with the temperature to which the sample was heated. Variations in resident time for which the protein was kept at an elevated temperature, in protein concentration or in the speed of heating and cooling did not affect dimer formation (Figure S9). 
The denaturation curves for the domain-swapped dimers show two major transitions (Figure 4A-B). The first unfolding transition occurs between $45^{\circ} \mathrm{C}$ and $65^{\circ} \mathrm{C}$, and is associated with a minor loss of helicity and partial exposure of hydrophobic residues as indicated by a red shift in the fluorescence spectrum. The corresponding heat capacity peak begins near the midpoint of the spectral denaturation curves at $52^{\circ} \mathrm{C}$ and ends at $65^{\circ} \mathrm{C}$ where spectroscopic signals plateau. Interestingly, this peak comprises of two transitions, most likely corresponding to the melting of two different dimers in the same temperature range. The second major transition follows the first one and coincides with the single unfolding transition of the monomer. Unfolding occurs between 65 and $78^{\circ} \mathrm{C}$, and is associated with further loss of ellipticity, extensive red shift and a single heat capacity peak. These results suggest that, with increasing temperature, the dimer undergoes structure "opening" followed by a collapse to a monomer-like structure which then proceeds to complete unfolding along the same pathway as a native monomer. This view is further supported by the analysis of oligomeric states after heating of the dimer to temperatures above the first transition point and subsequent cooling. This procedure leads to the formation of functional monomers (Figure S8B). Kinetics of the dimer unfolding measured using $\mathrm{CD}$ spectroscopy in the $58-70^{\circ} \mathrm{C}$ range showed triphasic behavior: (i) fast decrease of ellipticity, (ii) slightly slower phase with the opposite direction of amplitude and (iii) very slow phase with small amplitude in the direction of the first phase (Figure 4C). The second phase is lost at temperatures above $67^{\circ} \mathrm{C}$ and the curves can be fitted with a double exponential curve. At this range, rates of the second phase correspond with the unfolding rates of the monomer (Figure S7C). Kinetic data are highly consistent with our interpretation of the temperature scanning experiments.

Furthermore, we fitted all data globally to a four-state model, a partially reversible unfolding mechanism, using Calfitter v1.3 (Figure 4D) ${ }^{29,30}$. The model provides an acceptable fit to the data and enables a robust estimation of the energy barriers of each unfolding transition (Figure S10). The largest deviation was observed for the first DSC peak since it includes transitions of two dimers which occur at similar temperatures. Since the dimers are in 1:1 ratio in solution, based on native PAGE analysis, their unfolding transitions can be resolved by fitting the first transition to the unfolding model with two parallel pathways (Figure 4B). The resulting average of $\Delta \mathrm{H}_{\mathrm{cal}}$ values for each dimer, 180 and $88 \mathrm{~kJ} / \mathrm{mol}$ (average $\sim 134 \mathrm{~kJ} / \mathrm{mol}$ ), corresponds well with the $\Delta \mathrm{H}_{\text {cal }}$ value of this transition from the global fit ( $\sim 133 \mathrm{~kJ} / \mathrm{mol})$. The second transition can be approximated with the Lumry-Eyring model, as with the monomer. We speculate that as the monomeric unit unfolds at high temperatures, the 
refolding rate becomes significantly slower compared to the rate of irreversible denaturation $(\mathrm{I} \rightarrow \mathrm{U})$, which results in an overestimation of the energy barrier of this step and poor resolution of the last unfolding step. The overall $\Delta \mathrm{H}_{\text {cal }}$ for the whole transition $(\sim 630 \mathrm{~kJ} / \mathrm{mol})$ corresponds well with the value obtained by global fitting of the monomer unfolding data ( $\sim 650 \mathrm{~kJ} / \mathrm{mol})$. We conclude that the collected data can be explained with this minimal unfolding model.

A

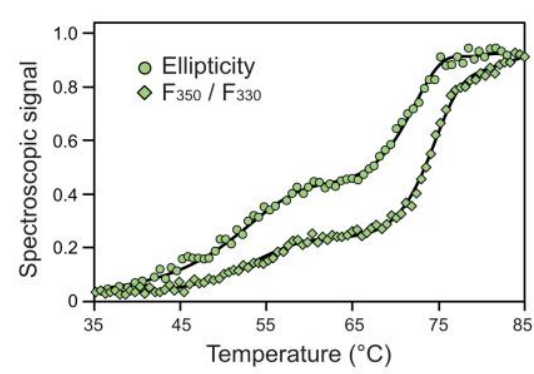

D

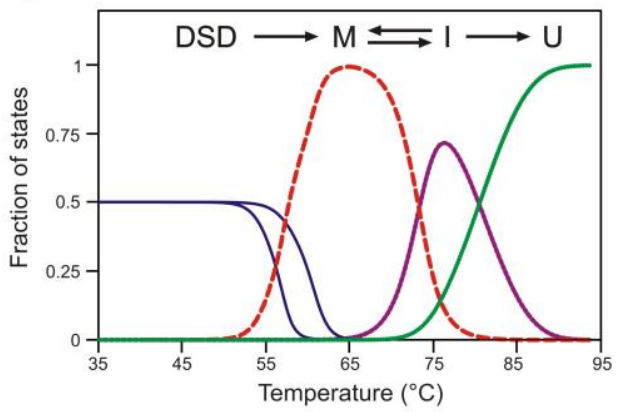

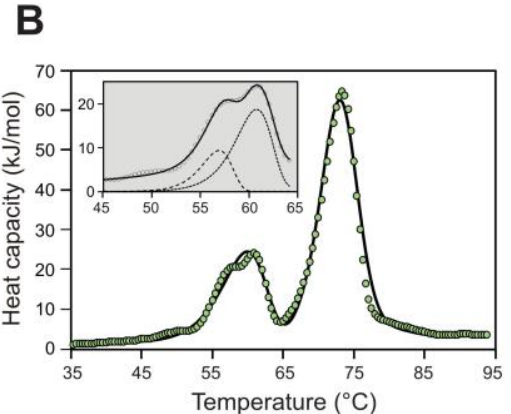

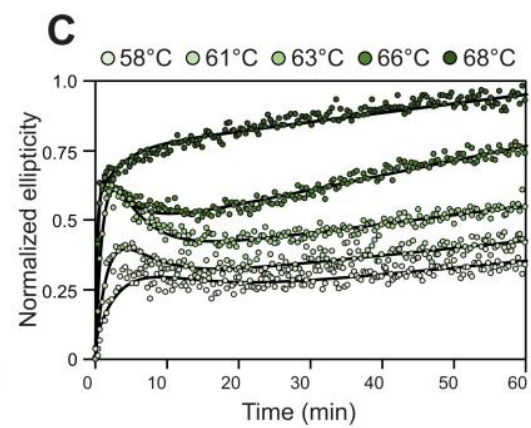

E

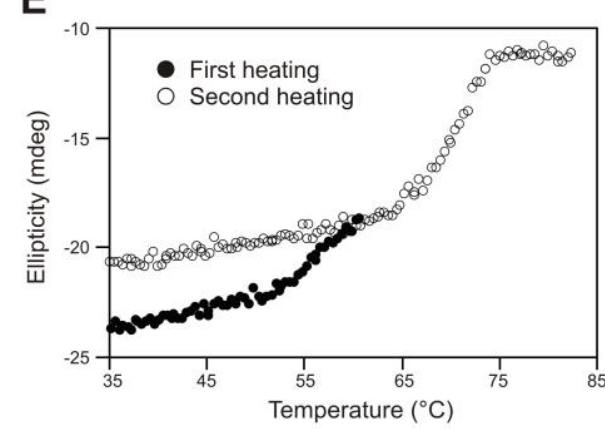

Figure 4. Global analysis of DhaA115 dimer unfolding. (A) Temperature scanning experiments carried out at $1{ }^{\circ} \mathrm{C} / \mathrm{min}$ scan rate followed by $\mathrm{CD}$ (circles) and fluorescence (diamonds) spectroscopies. Ellipticity at $224 \mathrm{~nm}$ and the ratio of fluorescence intensities at 350 and $330 \mathrm{~nm}$ are plotted against the temperature. (B) Differential scanning calorimetry thermograph. The inset presents deconvolution of the individual dimer dissociation. (C) Unfolding kinetics measured by monitoring changes of ellipticity at $224 \mathrm{~nm}$ at different temperatures. (D) The fraction of states calculated from the global fitting (black lines in all graphs) as a function of temperature: DSD - domain-swapped dimers (blue), M - monomer (red), I - intermediate (purple), U - unfolded state (green). (E) Reheating experiment of DhaA115 dimers measured by monitoring changes in ellipticity at $224 \mathrm{~nm}$. The sample was first heated to $60^{\circ} \mathrm{C}$ (black circles), then cooled down to $25^{\circ} \mathrm{C}$, and finally heated to $80^{\circ} \mathrm{C}$ (open circles) at $1{ }^{\circ} \mathrm{C} / \mathrm{min}$ scan rate.

Refolded Monomers Cannot Form Domain-Swapped Dimers. Similar results were obtained from chemical denaturation of DhaA115. Unfolding of DhaA115 using urea is more potent and leads to a completely unfolded polypeptide chain, in contrast to the heat-denatured 
state which retains a residual structure (Figure S11). Denaturation curves of both monomer and dimers, plotted as ellipticity at $224 \mathrm{~nm}$ and an average emission wavelength of fluorescence spectra, each show two transitions and can be fitted using a three-state unfolding model (Figure S12). The unfolding curves of both oligomeric states nearly perfectly overlap with the midpoints around 4 and $6 \mathrm{M}$ urea. This indicates that the dimer first splits to monomer-like structures at low urea concentration and then unfolds along the same pathway as a native monomer. Analysis of oligomeric states in the presence of 0 to $3 \mathrm{M}$ urea using the native PAGE confirmed this interpretation, showing gradual loss of bands corresponding to dimers and a simultaneous increase in the monomeric fraction with its midpoint around $2.2 \mathrm{M}$ urea (Figure S13, Figure S12A). Refolding of the DhaA115 was achieved by dilution or dialysis of the partially or completely denatured sample from 5 and $8 \mathrm{M}$ urea, respectively. In all cases, refolding yielded a mixture of native-like monomers and misfolded aggregates at all protein concentrations tested (Figure S14). Precipitation occurred at a protein concentration above $1 \mathrm{mg} \cdot \mathrm{mL}^{-1}$. The ratio between aggregates and monomer was concentration-dependent. However, no dimer formation was observed during any of these unfolding/refolding experiments.

Structure-Based Mutagenesis Reveals Importance of Mutations in the Hinge. Next, we questioned whether the domain swapping is induced primarily by the mutations in the hinge regions or those at the secondary interfaces. Based on the structure of the DhaA115DSD1 dimer, we first introduced three mutations into wild-type DhaA, to create DhaA176 $(\mathrm{T} 148 \mathrm{~L}+\mathrm{A} 172 \mathrm{I}+\mathrm{C} 176 \mathrm{~F})$. The effect of mutations based on DhaA115-DSD2 was tested in two-point mutant DhaA178 (E20S+D198W). In the second round of mutagenesis, the stabilizing mutation A155P was inserted into both of these mutants, yielding the four-point mutant DhaA177 (T148L+A155P+A172I+C176F) and the three-point mutant DhaA179 $(\mathrm{E} 20 \mathrm{~S}+\mathrm{A} 155 \mathrm{P}+\mathrm{D} 198 \mathrm{~W})$. All newly constructed mutants were expressed and purified as soluble proteins, and their proper folding confirmed with CD spectroscopy (Figure S15). Analysis of oligomeric species content confirmed that all four variants form dimeric forms (Figure S15B), although much less abundantly than DhaA115. Despite the intensive effort, we could only obtain well-diffracting crystals for the dimeric fraction of DhaA177, which is the variant with the highest fraction of oligomeric species among the four additional constructs.

The DhaA177 crystals belong to the $P 2{ }_{1} 2{ }_{1} 2_{1}$ space group, and its structure was solved by molecular replacement at $2.55 \AA$ resolution (Table S2). The asymmetric unit is formed by 
four polypeptide chains which are assembled into two non-crystallographic domain-swapped dimers. To our surprise, these domain-swapped dimers, hereafter referenced as DhaA177DSD3, revealed a third, completely novel mode of domain swapping. In this conformation, the short L10 loop $\left(\mathrm{P}_{155} \mathrm{D}\right)$, connecting the $\alpha 4$ and $\alpha 5^{\prime}$ cap helices, functions as a hinge (Figure 5A-B). The residue P155, introduced by the computational design as a stabilizing mutation $^{5}$, plays a central role in the hinge region of DhaA177-DSD3. No apparent stabilizing secondary interface is present in the structure of DhaA177-DSD3 and all physical contacts between the two catalytic units are entirely mediated through the hinge region (Figure 5C).

Inspection of crystallographic packing revealed that the DhaA177 domain-swapped dimers are tightly packed into a Z-shaped tetramer through an extensive dimer-to-dimer interface (Figure 5D-E). This interface is built around the side chains of two F144 residues from different dimers which are packed against each other. Amino acid composition of the dimer-to-dimer interface in DhaA177-DSD3 resembles the secondary interface observed in the DhaA115-DSD1 structure (Figure 2A). Expansion along the crystallographic two-fold screw axis reveals that the DhaA177 tetramers, which are composed of two domain-swapped dimers, are repeated building blocks of crystallographic open-ended filament-like structures in the corresponding crystal lattice (Figure 5F).

The mutagenesis experiments showed that four stabilizing mutations are sufficient to induce domain-swapping in the DhaA enzyme. Topological comparison of all three types of observed DhaA domain-swapped dimers is shown in Figure 6. In all three types of DhaA domain-swapped dimers, the swapping is mediated by a hinge loop located in the cap domain, as visualized in a morphing movie (Movie 1). A conformationally malleable cap domain shapes the morphology of internal enzyme access tunnels, which are known determinants of catalytic properties for this enzyme family. ${ }^{31,32}$ We therefore hypothesized that the domainswapped species could exhibit novel catalytic properties. 
A

DhaA177 protomer type-3

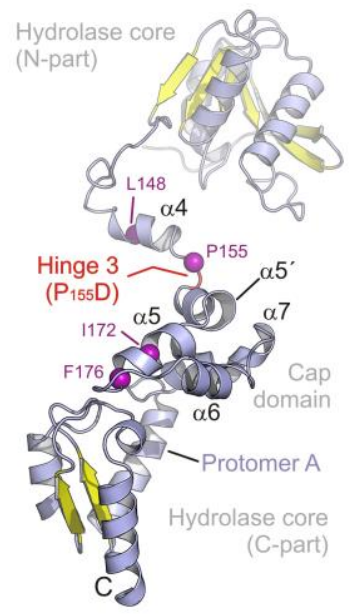

B

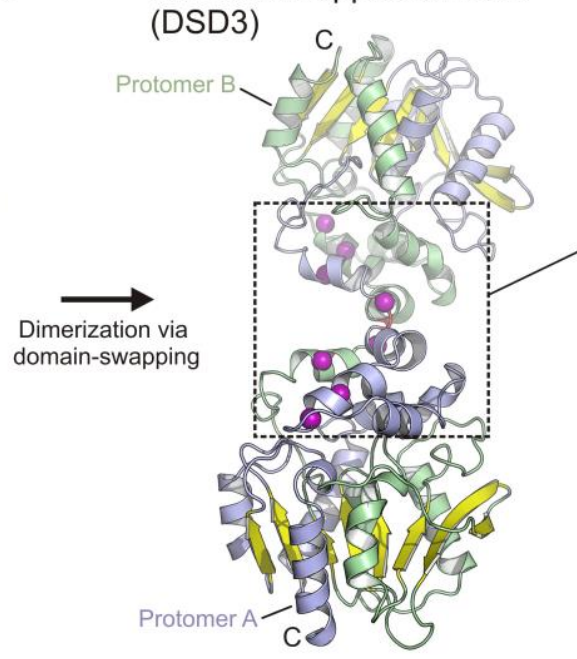

C Close-up of hinge 3 region

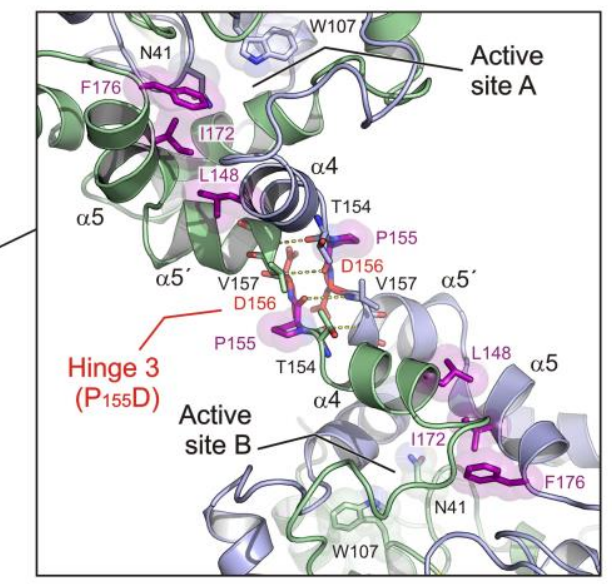

E Dimer-to-dimer molecular interface

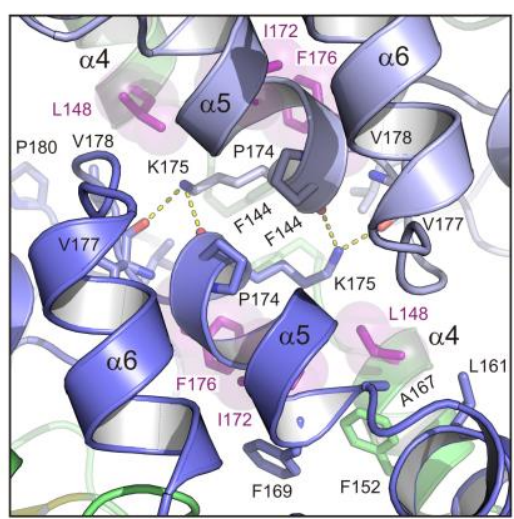

$\mathbf{F}$ Dimer-to-dimer crystal packing (Z-shaped tetramer)

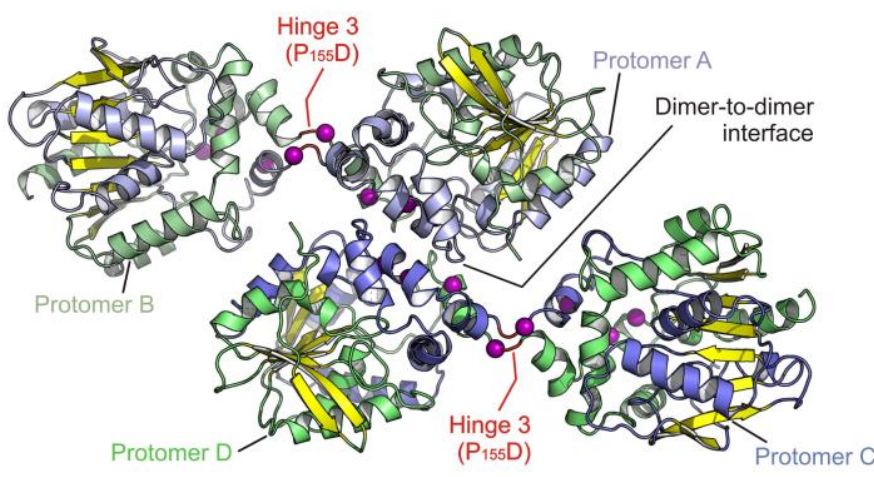

D Protomer C Crystallographic open-ended filament-like structure

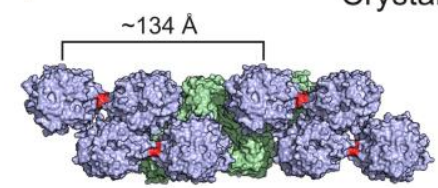

Side view
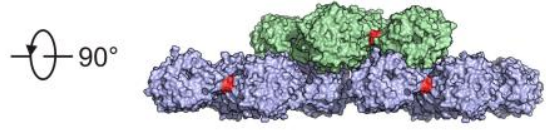

Top view

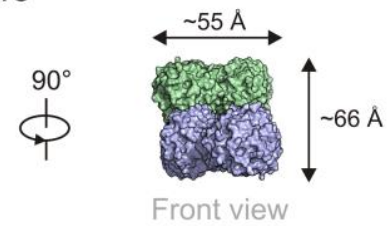

Front view

Figure 5. X-ray structure of domain-swapped dimer DhaA177-DSD3. Cartoon representations of (A) DhaA177 protomer type-3, (B) DhaA177-DSD3 dimer, (C) close-up view of the hinge 3 region, (D) dimer-to-dimer crystal packing, (E) close-up view of the dimer-to-dimer molecular interface, and (F) surface representation of crystallographic open-ended filament-like DhaA177 structure. The designed stabilizing mutations are depicted as purple spheres; the hinge 3 region $\left(\mathrm{P}_{155} \mathrm{D}\right)$ is coloured in red. The key residues stabilizing the intertwined protomers are shown as sticks; hydrogen bonds are shown as yellow dashed lines. The colour coding is the same as in Figure 2. 
A

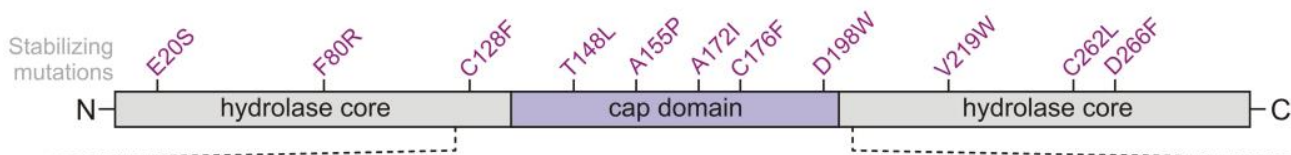

B

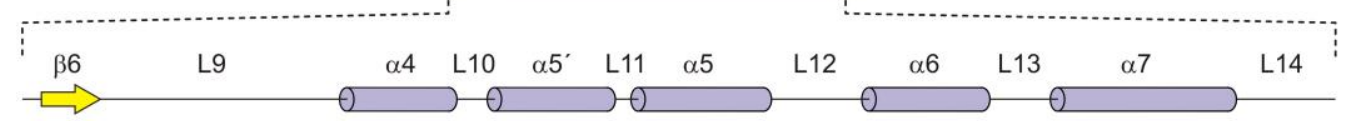

DhaA 125 G I ACMEF IRP I PTWDEWPEFARETFQAFRTADVGRELI IDQNAF I EGALPKCVVRPLTEVEMDHYREPFLKPVDREPLWRFPNELP IAGEP 215 Hinge 1

DhaA115-DSD1 125 G I AFMEF I RP I P TWDEWPEFARELFQAFRTPDVGREL I IDQNAF I EG ILPKFVVRPLTEVEMDHYREPFLKPVWREPLWRFPNELP IAGEP 215
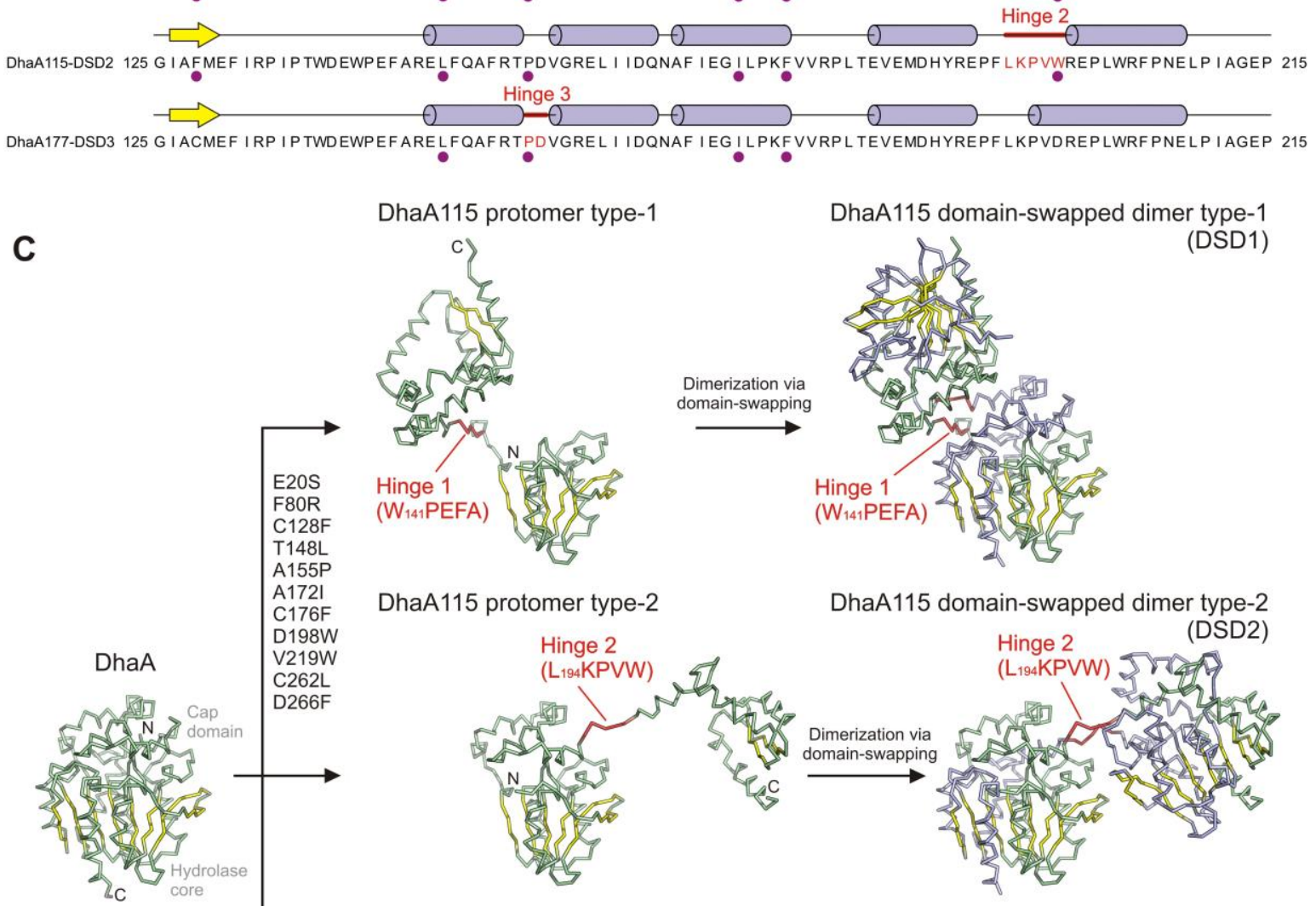

DhaA177 protomer type-3

DhaA177 domain-swapped dimer type-3
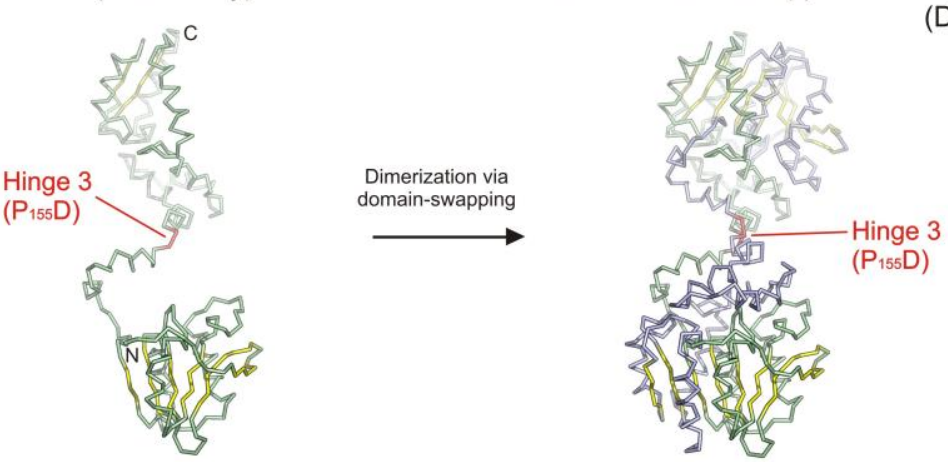

(DSD3)

Figure 6. The structural comparison of three different domain-swapping topologies. (A) Schematic representation of the protein sequence showing the domain topology of DhaA115 and the positions of the stabilizing mutations. (B) Partial structure-based alignment of DhaA, DhaA115 and DhaA177 sequences. The stabilizing mutations are highlighted with violet dots. The secondary structure elements are shown above every sequence. The hinge regions are coloured red. (C) Ribbon 
representations of DhaA monomeric structure along with three different domain-swapped dimers DhaA115-DSD1, DhaA115-DSD2 and DhaA177. The colour coding is the same as in Figure 2.

\section{See attached .mp4 file}

Movie 1. Putative conformational interconversions between the DhaA swapped-dimers. The morph was generated between crystal structures DhaA115-DSD (PDB ID 6TY71), DhaA115-DSD2 (PDB ID 6XT8) and DhaA177-DSD3 (PDB ID 6XTC) using PyMol (Schrödinger, LLC). Note that all conformational changes are occurring in the cap domain, while the $\alpha / \beta$-hydrolase core is compact during the topological transitions.

Domain-Swapped Dimers Exhibit Enhanced Catalytic Efficiency. Due to the reduction of enzyme access tunnels ${ }^{26}$, the catalytic activity of DhaA115 at ambient temperature was diminished compared to the wild-type DhaA. ${ }^{27}$ To find out how the domain swapping affects catalysis, the dehalogenase activities of the monomeric and dimeric DhaA115 forms were assayed with five halogenated substrates. Surprisingly, the activity of the DhaA115 dimeric form with all tested substrates increased 3.5 to 8 times, compared to the monomeric counterpart at $37^{\circ} \mathrm{C}$ (Figure $7 \mathrm{~A}$ ). To determine whether the several-fold improvement in dehalogenase activity was not merely the result of a temperature optimum shift, we compared the dehalogenase activity of engineered DhaA115 and DhaA177 over a wide range of temperatures. The temperature profiles of activities with 1,2-dibromoethane were shifted to lower temperatures for both DhaA115 and DhaA177 dimers, compared to their monomeric counterparts (Figure 7B-C). However, the catalytic activities at their temperature maxima showed that the domain-swapped dimer of DhaA115 is at least 5.5 times more active than its corresponding monomer, and the domain-swapped dimer of DhaA177 is 3.8 times more active (Figure 7B-C).

The activity-temperature profiles of the dimers have two peaks at temperatures that correlate with the onset temperatures of the dimers' dissociation and subsequent unfolding transitions observed in the temperature scanning experiments (Figure 7B-C). Moreover, the catalytic activities of DhaA115 and DhaA177 dimers at temperatures above the first transition (where we assume their dissociation occurs) are strikingly similar to those of the native monomers. These observations are further supported by the steady-state kinetics measurements of monomeric and dimeric fractions of DhaA115 at different temperatures 
(Figure 7D-F). The catalytic efficiency of the dimeric fraction of DhaA115 $\left(k_{\text {cat }} / K_{\mathrm{M}}=368.83 \mathrm{~s}^{-}\right.$ $\left.{ }^{1} \cdot \mathrm{mM}^{-1}\right)$ is 39 times higher than that of the monomer $\left(k_{\mathrm{cat}} / K_{\mathrm{M}}=9.33 \mathrm{~s}^{-1} \cdot \mathrm{mM}^{-1}\right)$ at $37^{\circ} \mathrm{C}$. At temperatures above $50^{\circ} \mathrm{C}$, the catalytic efficiency of the dimers is only twice as high, compared to the monomer (Figure 7F).

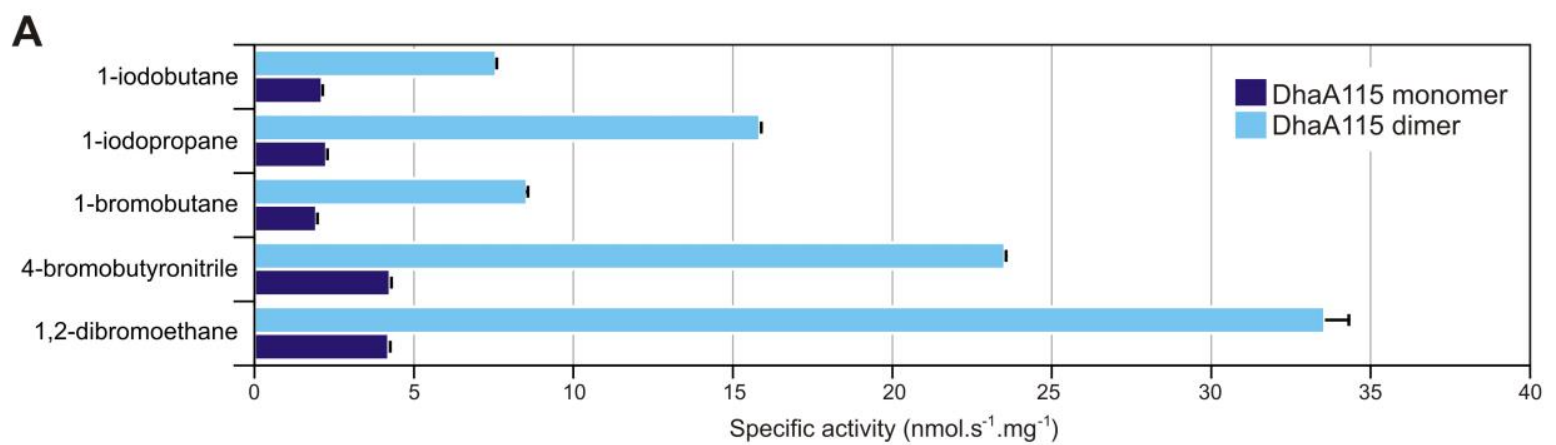

B

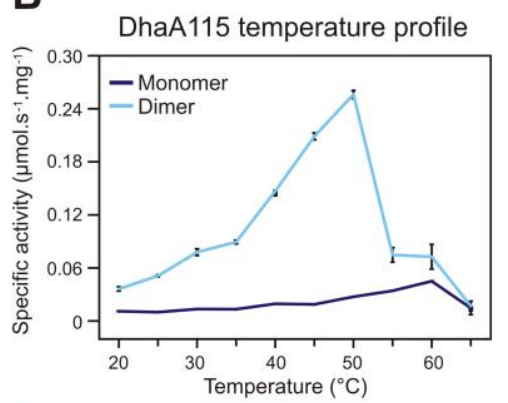

C

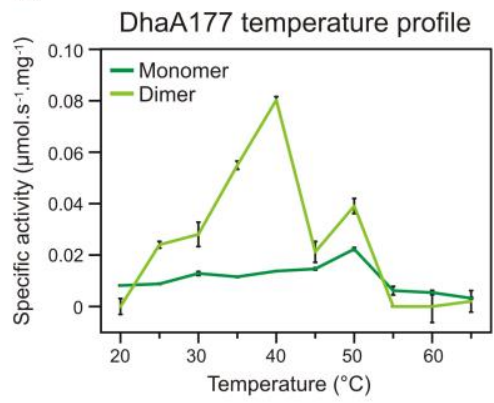

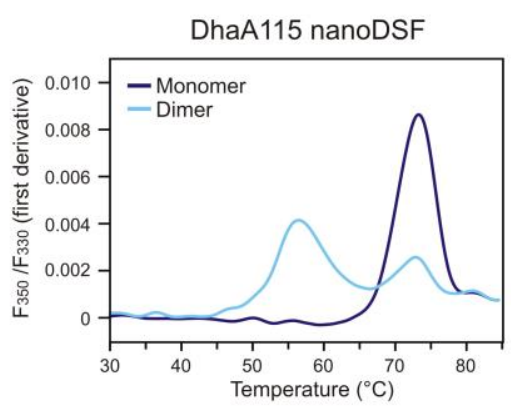

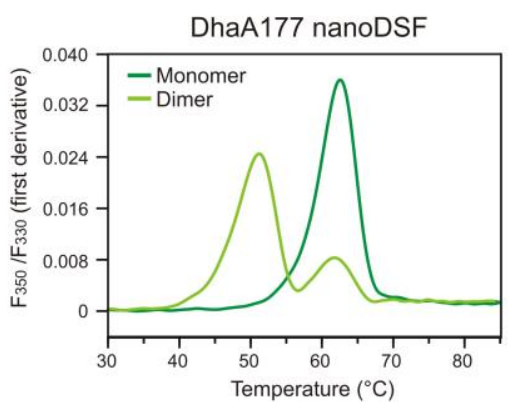

D

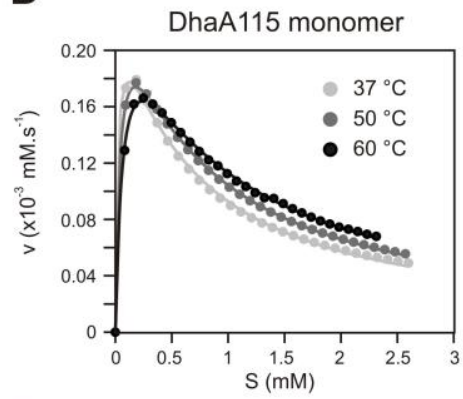

$\mathbf{E}$

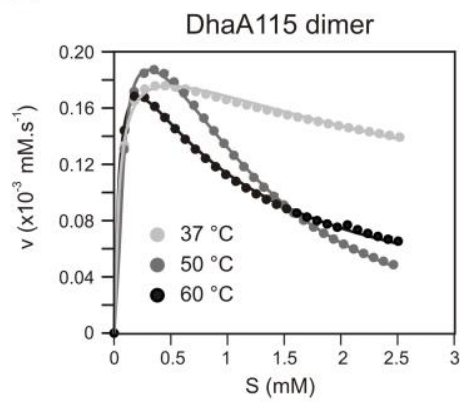

$\mathbf{F}$

\begin{tabular}{lcccccc}
\hline Enzyme & $\mathrm{T}\left[{ }^{\circ} \mathrm{C}\right]$ & $\mathrm{k}_{\text {cat }}\left[\mathrm{s}^{-1}\right]$ & $\mathrm{K}_{\mathrm{M}}[\mathrm{mM}]$ & $\mathrm{K}_{\mathrm{sI}}[\mathrm{mM}]$ & $\mathrm{n}$ & $\mathrm{K}_{\text {cat }} / \mathrm{K}_{\mathrm{M}}$ \\
\hline DhaA115 & 37 & $0.28 \pm 0.02$ & $0.03 \pm 0.001$ & $0.58 \pm 0.02$ & $-^{\mathrm{a}}$ & $9.33 \pm 0.74$ \\
monomer & 50 & $9.88 \pm 0.27$ & $0.05 \pm 0.002$ & $0.66 \pm 0.01$ & $-^{\mathrm{a}}$ & $208.35 \pm 10.17$ \\
& 60 & $22.66 \pm 0.38$ & $0.07 \pm 0.003$ & $0.82 \pm 0.04$ & $-{ }^{\mathrm{a}}$ & $315.23 \pm 15.65$ \\
\hline \multirow{2}{*}{ DhaA115 } & 37 & $15.86 \pm 0.76$ & $0.04 \pm 0.002$ & $5.56 \pm 0.23$ & $-^{\mathrm{a}}$ & $368.83 \pm 26.43$ \\
dimer & 50 & $31.89 \pm 0.29$ & $0.07 \pm 0.002$ & $1.71 \pm 0.08$ & $1.95 \pm 0.02$ & $441.66 \pm 11.62$ \\
& 60 & $37.76 \pm 0.01$ & $0.06 \pm 0.001$ & $0.83 \pm 0.03$ & $-{ }^{\mathrm{a}}$ & $679.73 \pm 14.73$ \\
\hline
\end{tabular}

a not applicable

Figure 7. Comparison of the catalytic properties of monomers and domain-swapped dimers. (A) Dehalogenase activity of DhaA115 monomeric and dimeric fractions towards five halogenated substrates. The activity was determined in glycine buffer at $37^{\circ} \mathrm{C}$ and $\mathrm{pH}$ 8.6. Presented are averages from three independent experiments, the error bars represent standard deviations. (B, C) Temperature 
profiles of monomeric and dimeric fractions of DhaA115 and DhaA177 with 1,2-dibromoethane (left panel) and temperature stability measurements using nanoDSF (right panel). (D, E) Measurements of steady-state kinetics of DhaA115 monomeric and dimeric fractions measured with 1,2-dibromoethane at $37^{\circ} \mathrm{C}, 50^{\circ} \mathrm{C}$ and $60^{\circ} \mathrm{C}$ in glycine buffer at $\mathrm{pH} 8.6$. The thin lines show the nonlinear regression fit of the particular kinetic model. (F) Steady-state kinetic parameters of DhaA115 monomeric and dimeric fractions under the conditions described in panels D and E. The standard deviations were calculated from three independent experiments.

Measured catalytic efficiencies have to be normalized to the number of active sites. This normalization results in nearly identical values for the monomer and dissociated dimers. The increase of substrate inhibition $\left(K_{\mathrm{SI}}\right)$ in the reactions catalyzed by DhaA115 dimers from $\sim 5.56 \mathrm{mM}$ at $37{ }^{\circ} \mathrm{C}$ to $\sim 0.83 \mathrm{mM}$ at $60{ }^{\circ} \mathrm{C}$, reaching the same level as observed for the monomer at the same temperature $(\sim 0.82 \mathrm{mM})$, provides additional experimental evidence for our observations that the domain-swapped dimers dissociate to functional monomeric subunits. The increased activity and catalytic efficiency of the dimers are the direct consequences of the unique structural arrangement of the polypeptide chains in the domainswapped conformation.

\section{DISCUSSION}

In this work, we provided direct experimental evidence that contemporary computer algorithms used for protein stabilization may affect protein folding. We previously designed hyperstable DhaA $115^{5,27}$ using force field calculations and evolutionary analysis with automated software FireProt ${ }^{5,33}$. A recent structural study carried out on the monomer of DhaA115 revealed an intricate network of molecular interactions that reinforce the engineered $\alpha \beta \alpha$ sandwich architecture. ${ }^{26}$ Mutations to bulky aromatic amino acids at the protein surface trigger long-distance backbone changes through multiple cooperative interactions. These interactions produce an unprecedented double-lock system that closes the molecular gates to the active site and reduces the volumes of the access tunnels ${ }^{26}$. Surprisingly, we have observed that this computer-aided stabilization of DhaA was accompanied by partial de novo oligomerization $^{27}$. 
Here, we showed that whilst the wild-type DhaA exists solely as a monomer, the engineered DhaA115 variant can additionally form dimers and higher oligomeric forms, when overexpressed in Escherichia coli. Crystallographic analysis of the DhaA115 dimeric form revealed two different dimer topologies, both formed by a so-called three-dimensional domain-swapping mechanism. The crystallographic structures of DhaA variants determined in this study are the first domain-swapped structures of haloalkane dehalogenases to be seen. Several complementary experiments ruled out the possibility of the structure as a crystallization artefact ${ }^{34}$. The SAXS and crosslinking MS experiments provided direct experimental evidence that the domain-swapped dimers exist in solution. Crucially, these structural observations raised a fundamental question: why does domain swapping occur during computer-aided stabilization of a protein?

Our findings demonstrate that the domain swapping in DhaA occurs through solventexposed loops - cryptic hinge regions - which are parts of the cap domain (Figure 8). Interestingly, stabilizing mutations are frequently found in these cryptic hinge loops, in their vicinity and/or in the secondary interfaces, where they contribute to the non-covalent interactions between the intertwined polypeptide chains. In the DhaA115-DSD1 structure, no engineered mutation is present in the hinge loop L9 $\left(\mathrm{W}_{141} \mathrm{PEFA}\right)$. However, the three designed mutations (T148L, A172I and C176F) take part in a novel hydrophobic patch that stabilizes the domain-swapped dimer (DSD1) topology through the newly formed secondary interface. In DhaA115-DSD2, the stabilizing mutation D198W is localized in the hinge loop L13 (L ${ }_{194} \mathrm{KPVW}$ ). Additionally, mutation E20S participates in the extended network of protein-solvent interactions that stabilize the secondary interface. In the DhaA177-DSD3 dimer, mutation $\mathrm{A} 155 \mathrm{P}$ is a part of the hinge region $\left(\mathrm{P}_{155} \mathrm{D}\right)$. It has been previously demonstrated that prolines are frequently found in the hinge loops ${ }^{35}$. Native or engineered proline residues are found in all three respective hinges of our domain-swapped dimers. The structural analyses of these dimers show that the putative stabilizing mutations designed by force field calculations not only stabilized the monomeric form ${ }^{26}$ but, at the same time, coincidentally increased domain-swapping propensity. Multi-point mutations to bulky hydrophobic and/or aromatic residues created a new hydrophobic interface, which is responsible for the co-translational misfolding. We conclude that the most detrimental misfolding effects can be caused when protein engineering efforts simultaneously: (i) manipulate the sequence of cryptic hinge regions and (ii) introduce new interaction interfaces 
that increase the domain-swapping propensity by lowering the free energy of the incorrectly folded (i.e. domain-swapped) intermediates.

Moreover, our study illustrates that the DhaA protein was evolutionarily optimized towards folding into the monomeric protein. A few mutations are sufficient to modify the energy landscape and lead to domain-swapped intermediates. Domain-swapped dimers are less thermodynamically stable than their monomeric counterparts, and functional native-like monomers can be restored by thermal dissociation at elevated temperatures. The average difference between the stability of monomers and dimers is $\sim 13^{\circ} \mathrm{C}$ of $T_{\mathrm{m}}{ }^{\text {app }}$. MD simulations suggest that the dimers are less stable due to a significant increase in their structural fluctuation. There is cumulative evidence that domain swapping played a key role in the emergence of oligomeric proteins during evolution ${ }^{36}$. There are also many examples describing how domain swapping is a detrimental process leading to protein misfolding and aggregation, associated with human pathologies ${ }^{23,37}$.

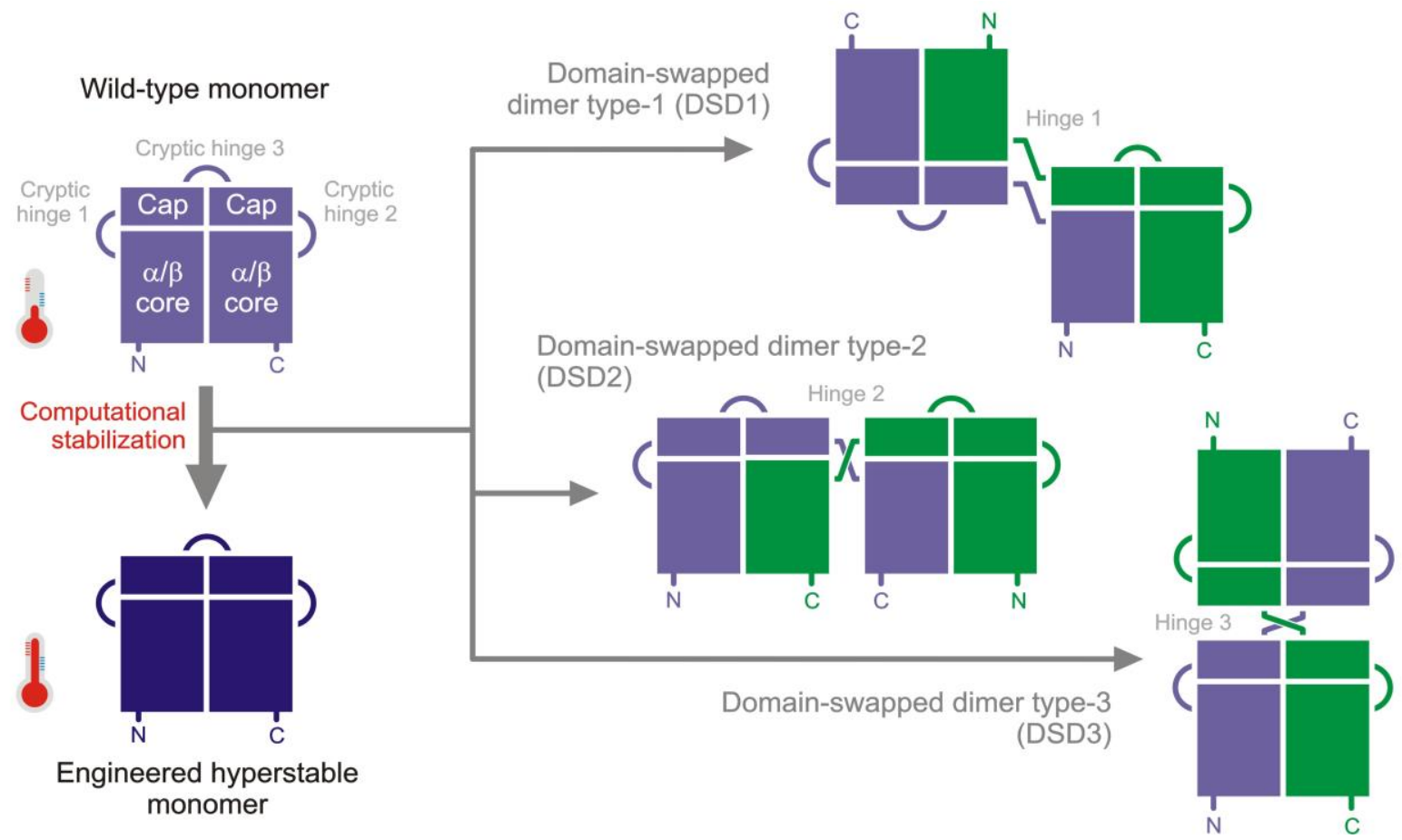

Figure 8. Schematic representation of computer-aided stabilization of $\alpha / \beta$-hydrolase fold DhaA enzyme that was unintendedly accompanied by the in-cell formation of catalytically-active domainswapped dimers.

Domain swapping of the DhaA115 dimer cannot be induced in vitro by refolding from denatured states. It possibly competes with monomer folding at low temperatures (low urea 
concentration) and formation of aggregates during refolding at high temperatures (high urea concentration). Formation of increasingly larger aggregates observed during refolding of the wild-type DhaA and DhaA115 indicate that aggregation could be caused by consecutive domain swapping. The domain swapping must occur in vivo during recombinant protein expression. We monitored the time course of protein expression in E. coli and were able to detect a fraction of dimer approximately 3.5 hours after expression induction. The fraction of dimer triples in the course of the next two hours and then gradually plateaus at $\sim 17-22 \%$ of the total DhaA115 after overnight expression. This suggests that the critical concentration of the recombinant protein must accumulate before domain swapping can occur, and that the crowding effect might play a role in this process. However, other effects such as the formation of an intermediate during co-translational folding, interaction with chaperones or other effects might be responsible for the domain swapping. Surprisingly, domain swapping significantly increased the catalytic efficiency of DhaA. This increase can be solely attributed to the inherent properties of these unique conformations.

\section{CONCLUSIONS}

We have elucidated the structural basis of the domain swapping mechanism of computationally stabilized DhaA by comprehensive biophysical, biochemical and computational analyses. The results provide a new view of the structure-function relationships of haloalkane dehalogenases and their folding landscapes. Our comprehensive study revealed hidden consequences for protein-folding through computational protein stabilization, which need to be taken into account when applying a rational stabilization to biomolecules of biological and pharmaceutical interest. In addition, our discovery highlights the importance of biophysical characterization techniques, for instance native nondenaturing PAGE, SEC, AUC and SAXS, that should always be applied during the control and quality checks of computationally designed proteins. Knowledge derived from this study can be exploited in future projects for the rational design of stable biocatalysts and for the study of folding, aggregation and stability of proteins.

\section{EXPERIMENTAL SECTION}


Protein Production and Purification. His-tagged DhaA variants were overexpressed from pET21b recombinant plasmids in Escherichia coli BL21(DE3). The expression was induced using $0.5 \mathrm{mM}$ IPTG at $20^{\circ} \mathrm{C}$ for 16 hours. The cells were harvested using centrifugation at $11,806 \mathrm{~g}$ at $4{ }^{\circ} \mathrm{C}$ for $10 \mathrm{~min}$. The pellet was re-suspended in purification buffer A (500 mM $\mathrm{NaCl}, 10 \mathrm{mM}$ imidazole, $20 \mathrm{mM}$ potassium phosphate buffer $\mathrm{pH}$ 7.5), and sonicated using Sonic Dismembrator Model 705 (Fisher Scientific, USA) in 3 cycles, each of 2 min (5s pulse $/ 5 \mathrm{~s}$ pause) with amplitude $50 \%$. Disrupted cells were centrifuged at $21,000 \mathrm{~g}$ at $4{ }^{\circ} \mathrm{C}$ for $1 \mathrm{~h}$. His-tagged proteins were purified on a Ni-chelating column (Ni-NTA Superflow cartridge) equilibrated with the purification buffer A. The column-bound enzymes were eluted with a gradient of purification buffer A supplemented with $300 \mathrm{mM}$ imidazole. The eluted proteins were further purified with size-exclusion chromatography using a HiLoad 16/600 Superdex 200 gel filtration column (GE Healthcare). Separated peak fractions were pooled and concentrated with an Amicon Ultra centrifugal filter unit (Merck Millipore Ltd), and protein concentrations were measured with a DeNovixR ${ }^{\circledR}$ DS-11 Spectrophotometer (DeNovix Inc., USA).

Crystallization, X-ray Data Collection and Structure Determination. Diffraction-quality crystals of DhaA115-DSD1 were obtained using the sitting-drop vapor diffusion method. Briefly, DhaA115 protein was dialyzed into $50 \mathrm{mM}$ Tris- $\mathrm{HCl}$ buffer (pH 7.5) and concentrated to $\sim 11.5 \mathrm{mg} \cdot \mathrm{mL}^{-1}$. Crystallization was set up using the sitting drop vapor diffusion method with Cryschem 24-well crystallization plates (Hampton Research) at $19^{\circ} \mathrm{C}$. After 4 to 8 days, crystals appeared in a mixture (1:1) of protein and crystallization buffer consisting of 0.1 M MES/imidazole pH 6.5, 10\% (w/v) PEG 20K, 20\% (v/v) PEG MME 550,

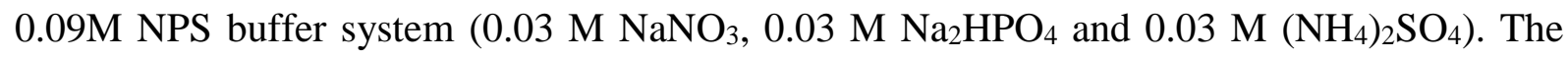
crystals obtained were directly flash-frozen in liquid nitrogen with no additional cryoprotection. X-ray frames of DhaA115-DSD1 were collected using the MX beamline I03 (Diamond Light Source, UK) at a wavelength of $0.97625 \AA$ with a Pilatus 6M-F detector. Diffracting crystals of DhaA115-DSD2 were analogously obtained using a vapor diffusion technique in a crystallization buffer containing $0.1 \mathrm{M}$ Bis-Tris ( $\mathrm{pH} 5.5), 0.2 \mathrm{M} \mathrm{NH}_{4} \mathrm{NO}_{3}$ and $16 \%$ PEG 3350. After 3 to 6 days at $19^{\circ} \mathrm{C}$, the crystals were harvested and flash-frozen in a reservoir solution supplemented with $20 \%$ glycerol. X-ray data for DhaA115-DSD2 were collected from the ESRF ID23-1 beamline ${ }^{38}$ (Grenoble, France) at a wavelength of $0.861 \AA$ using a Pilatus 6M detector. Crystals of DhaA177-DSD3 were also obtained using a vapor 
diffusion technique. Diffracting crystals of DhaA177 were obtained from a crystallization buffer consisting of $0.1 \mathrm{M}$ Tris $(\mathrm{pH} 8.5), 0.2 \mathrm{M} \mathrm{LiSO}_{4}$ and $1.26 \mathrm{M}\left(\mathrm{NH}_{4}\right)_{2} \mathrm{SO}_{4}$. After 3 to 9 days at $19^{\circ} \mathrm{C}$, the crystals were harvested and flash-frozen in a reservoir solution supplemented with $20 \%$ glycerol. X-ray frames of DhaA177-DSD3 were collected from the PX3 beamline at SLS (Paul Scherrer Institute, Switzerland) at a wavelength of $0.99987 \AA$ using a Pilatus 2M-F detector. The crystallographic data were processed using $\operatorname{XDS}^{39}$ and Aimless ${ }^{40}$. Initial phases were solved by molecular replacement using Phaser $^{41}$ software implemented in the Phenix package ${ }^{42}$. The X-ray structure of wild-type DhaA (PDB: 4HZG ${ }^{43}$ ) was used as a search model for molecular replacement during structure determination. The structure refinements were carried out over several cycles of automated refinement by the phenix.refine ${ }^{44}$ program, with manual model building carried out in $\operatorname{Coot}^{45}$. The final models were validated using tools provided by $\mathrm{Coot}^{45}$ and Molprobity ${ }^{46}$. Graphical visualizations of structural data were created using PyMOL ${ }^{47}$. Structural superposition was carried out using the secondary structure matching (SSM) superimpose tool in the $\operatorname{Coot}^{48}$. Atomic coordinates and structure factors of the DhaA115-DSD1, DhA115-DSD2 and DhaA177 domain-swapped enzyme variants were saved in the Protein Data Bank (www.wwpdb.org) ${ }^{49}$ under the PDB codes 6TY7, 6XT8 and 6XTC.

Semi-Analytical Size-Exclusion Chromatography. Protein samples eluted with $300 \mathrm{mM}$ imidazole (60\% gradient) using metal-affinity chromatography were loaded on the FPLC system ÄKTA Purifier (GE Healthcare) equipped with a UV 280 detection system and a Superdex 200 Increase 10/300 GL gel filtration column, equilibrated with $50 \mathrm{mM}$ phosphate buffer ( $\mathrm{pH}$ 7.5). Elution was carried out using the same buffer at a constant flow rate of 0.8 $\mathrm{mL} \cdot \mathrm{min}^{-1}$. The contents of oligomeric fractions were evaluated by peak integration area.

Analytical Size-Exclusion Chromatography. The quaternary structure was analyzed using an analytical size exclusion chromatography (SEC) system equipped with static light scattering, refractive index, ultraviolet and differential viscometer detectors. The system was calibrated by using bovine serum albumin as a protein standard. The Viscotec 305 TDA instrument (Malvern, UK) and the column Zenix-C 300 (Sepax Technologies, USA) were equilibrated by $50 \mathrm{mM}$ phosphate buffer $\mathrm{pH}$ 7.5. The protein, eluted with $300 \mathrm{mM}$ imidazole during affinity chromatography, was dialyzed overnight in $50 \mathrm{mM}$ phosphate buffer $\mathrm{pH} 7.5$, concentrated to $2.24,4.80$ and $7.67 \mathrm{mg} \cdot \mathrm{mL}^{-1}$, injected into the column and separated at a 
constant flow rate $0.3 \mathrm{~mL} \cdot \mathrm{min}^{-1}$ of the elution buffer. Retention volumes, molecular weights, hydrodynamic radius and intrinsic viscosities were evaluated using OmniSec software (Malvern, UK).

Analytical Ultracentrifugation. Analytical ultracentrifugation (AUC) experiments were carried out using a ProteomeLab XL-I analytical ultracentrifuge (Beckman Coulter, Indianapolis, IN, USA) equipped with an An-60 Ti rotor. Sedimentation velocity experiments were carried out in titanium double-sector centerpiece cells (Nanolytics Instruments, Potsdam, Germany) loaded with $390 \mu \mathrm{L}$ of both protein sample and reference solution (100 $\mathrm{mM} \mathrm{NaCl}$, $20 \mathrm{mM}$ potassium phosphate buffer $\mathrm{pH}=7.5$ ). DhaA115 samples, pre-incubated with different concentrations $\left(0.096,0.31\right.$ and $\left.0.68 \mathrm{mg} \cdot \mathrm{mL}^{-1}\right)$, were analyzed as part of the sedimentation velocity experiment. Data were collected using absorbance and interference optics at $20{ }^{\circ} \mathrm{C}$ at a rotor speed of $45,000 \mathrm{rpm}$. Scans were collected at $280 \mathrm{~nm}$ at $5 \mathrm{~min}$ intervals and $0.003 \mathrm{~cm}$ spatial resolution in continuous scan mode, with an interference laser duration of $0.1^{\circ}$. The partial specific volume of the protein, and the solvent density and viscosity were calculated from the amino acid sequence and buffer composition, respectively, using the software Sednterp (http://bitcwiki.sr.unh.edu). The data were analyzed with the continuous $c(s)$ distribution model implemented in the program Sedfit $15.01 b^{50}$. For the regularization procedure, a confidence level of 0.95 was used. The plots of $\mathrm{c}(\mathrm{s})$ distributions were created in GUSSI 1.3.1 ${ }^{51}$.

Native Polyacrylamide Gel Electrophoresis. The protein sample $\left(\sim 1 \mathrm{mg} \cdot \mathrm{mL}^{-1}\right)$ was mixed with loading buffer (35\% glycerol, $0.25 \mathrm{M}$ Tris- $\mathrm{HCl} \mathrm{pH}$ 6.8, 0.04\% Bromophenol Blue) in a ratio of $1: 3$ and $13 \mu \mathrm{L}$ of the mix was loaded into the $12.5 \%$ native gel with a $4 \%$ stacking gel layer. Electrophoresis was carried out in a Tris-glycine electrophoretic buffer pH 8.3 (25 mM Tris, $192 \mathrm{mM}$ Glycine) at $110 \mathrm{~V}$ and $4{ }^{\circ} \mathrm{C}$. Protein bands of polyacrylamide gels were stained with InstantBlue Protein Stain (Sigma), following the supplier's protocol, and checked by GS800 Calibrated Densitometer.

Circular dichroism (CD). The spectra were recorded at room temperature using a Chirascan spectrometer (Applied Photophysics, UK). Some $300-400 \mu \mathrm{L}$ of a protein sample at a concentration of $\sim 0.3 \mathrm{mg} \cdot \mathrm{mL}^{-1}$, dialyzed against $50 \mathrm{mM}$ phosphate buffer (pH 7.5), was 
placed in a $0.1 \mathrm{~cm}$ quartz cuvette and data were collected from 185 to $260 \mathrm{~nm}$, at $100 \mathrm{~nm} . \mathrm{min}^{-}$ ${ }^{1}, 1 \mathrm{~s}$ response time and $2 \mathrm{~nm}$ bandwidth. The final spectrum was an average of three individual scans that had been corrected for baseline noise.

Differential Scanning Fluorimetry (DSF). The thermal stability of enzyme variants was determined with label-free nanoDSF using a Prometheus NT.48 instrument (NanoTemper Technologies, Germany), which uses inherent tryptophan fluorescence to monitor protein unfolding. Capillaries were filled with protein samples $\left(\sim 1 \mathrm{mg} \cdot \mathrm{mL}^{-1}\right)$ in $50 \mathrm{mM}$ potassium phosphate buffer ( $\mathrm{pH} 7.5$ ) and fluorescence was monitored in the temperature range of 20-90 ${ }^{\circ} \mathrm{C}$ with $1{ }^{\circ} \mathrm{C} . \mathrm{min}^{-1}$ heating rate. Melting temperatures were determined from the ratio of tryptophan emission at 330 and $350 \mathrm{~nm}$ using PR-ThermControl (NanoTemper Technologies, Germany).

Dehalogenase Activity Measurements. Dehalogenase activity was measured using the colorimetric method described by Iwasaki et al. ${ }^{52}$ Dehalogenase reaction was tested on five halogenated substrates, namely 1,2-dibromoethane, 4-bromobutyronitrile, 1-bromobutane, 1iodopropane and 1-iodobutane, in 25-mL Reacti Flasks closed with Mininert Valves. Reaction mixtures were composed of $10 \mathrm{~mL}$ glycine buffer $(\mathrm{pH} \mathrm{8.6)}$ and $10 \mu \mathrm{L}$ of a substrate. The mixtures were incubated at $37^{\circ} \mathrm{C}$ in a shaking bath for 20 minutes. The reaction was initiated by addition of $150-600 \mu \mathrm{L}$ enzyme at a concentration of $0.4-1.2 \mathrm{mg} \cdot \mathrm{mL}^{-1}$. Reactions were monitored by withdrawing $1 \mathrm{~mL}$ aliquots at regular intervals, and stopped by addition of 100 $\mu \mathrm{L}$ of $35 \%$ nitric acid. Halide ions released by the dehalogenase reaction were measured spectrophotometrically at $460 \mathrm{~nm}$ after adding $100 \mu \mathrm{L}$ of Iwasaki solution I (28.4 mM $\mathrm{Hg}(\mathrm{SCN})_{2}$ in ethanol) and $200 \mu \mathrm{L}$ of Iwasaki solution II $\left(0.56 \mathrm{M} \mathrm{FeNH}_{4}\left(\mathrm{SO}_{4}\right)_{2} .12 \mathrm{H}_{2} \mathrm{O}, 21 \%\right.$ $\mathrm{HNO}_{3}$ ). Enzyme activity was quantified from the slope of the relationship between the product concentration and the time.

Temperature Profile Measurements. The specific activities of individual enzyme variants with 1,2-dibromoethane were assayed from $20^{\circ} \mathrm{C}$ to $65^{\circ} \mathrm{C}$ in 5 degree increments. The temperature profiles were measured using a capillary-based droplet microfluidic platform ${ }^{53}$ which enables characterization of enzymatic activity in droplets for multiple enzymes in one run. Briefly, the droplets were generated using Mitos Dropix (Dolomite, UK). A custom 
sequence of droplets (150 $\mathrm{nL}$ aqueous phase, $300 \mathrm{~nL}$ oil spacing) was generated by means of negative pressure (microfluidic pump) and the droplets were guided through a polythene tubing to the incubation chamber. Within the incubation chamber, the halogenated substrate was delivered to the droplets with a combination of microdialysis and partitioning between the oil (FC 40) and the aqueous phase. The reaction solution consisted of a weak buffer (1 mM HEPES, $20 \mathrm{mM} \mathrm{Na} \mathrm{SO}_{4}, \mathrm{pH}$ 8.0) and a complementary fluorescent indicator 8hydroxypyrene-1,3,6-trisulfonic acid (50 $\mu \mathrm{M}$ HPTS). The fluorescence signal was detected using an optical setup with an excitation laser $(450 \mathrm{~nm})$, a dichroic mirror with a cut-off at $490 \mathrm{~nm}$ filtering the excitation light and a silicon detector. By using a $\mathrm{pH}$-based fluorescence assay, small changes in the $\mathrm{pH}$ could be detected which enabled monitoring of the enzymatic activity. Reaction progress was analyzed as an end-point measurement recorded after passing through the incubation chamber. The reaction time was determined precisely for each data collection activity, and was approx. 4 min. The raw signal was processed with a droplet detection script written in MATLAB 2017b (Mathworks, USA).

Steady-State Kinetics. The steady-state kinetics of the DhaA115 monomer and dimer on 1,2dibromoethane were measured using a VP-ITC isothermal titration calorimeter (MicroCal, USA). A microcalorimeter reaction mixture vessel was filled with $1.4 \mathrm{~mL}$ of the enzyme at a concentration of $0.087-0.87 \mu \mathrm{M}\left(0.003-0.03 \mathrm{mg} \cdot \mathrm{mL}^{-1}\right)$. The substrate solution was prepared by dissolving $10 \mu \mathrm{L}$ of 1,2-dibromoethane in $4 \mathrm{~mL}$ of the $100 \mathrm{mM}$ glycine buffer ( $\mathrm{pH} 8.6$ ); its final concentration was verified with gas chromatography (Agilent, USA) for each measurement (approx. 12-13 mM). The substrate was titrated in $10 \mu 1$ injections with $150 \mathrm{~s}$ intervals into the reaction mixture vessel. Each injection increased the substrate concentration, leading to a change in the reaction rate (a change of heat generated) until the enzymatic reaction was saturated. A total of 28 injections were made during titration. The amount of heat released during conversion of $1 \mathrm{mM}$ substrate was determined in a separate experiment in which $10 \mu \mathrm{L}$ of the substrate were injected into a reaction vessel filled with $1.4 \mathrm{~mL}$ of enzyme and the heat released during total substrate conversion was measured. The final value was obtained as the average of the integrated area from 4 injections. The reaction rates reached after every injection (in units of thermal power) were recalculated to the enzyme turnover using the value from the total conversion experiment described above. The calculated enzyme turnover plotted against the actual concentration of the substrate after every injection was then 
fitted by nonlinear regression to kinetic models using Origin 6.1 (OriginLab, USA). The kinetic data for both the DhaA115 monomer and dimer measured at all temperatures were analyzed with equation (1), which describes substrate inhibition of the enzyme. The exception was the reaction of DhaA115 dimer at $50^{\circ} \mathrm{C}$ where equation (2), describing substrate inhibition with positive cooperative substrate binding, was used for the analysis.

(1)

$v_{0}=\frac{v_{\text {lim }}[S]}{K_{M}+[S]\left(1+\frac{[S]}{K_{S i}}\right)}$

(2)

$v_{0}=\frac{v_{l i m}[S]^{n}}{K_{M}^{n}+[S]^{n}\left(1+\frac{[S]^{n}}{K_{S i}}\right)}$

Small-Angle X-ray Scattering (SAXS). The SAXS datasets were collected using the BioSAXS-1000, Rigaku at CEITEC (Brno, Czech Republic). Data were collected at $293 \mathrm{~K}$ with a focused (confocal OptiSAXS optic, Rigaku) $\mathrm{Cu} \mathrm{K \alpha}$ X-ray (1.54 $\AA$ ). The sample to detector (PILATUS 100K, Dectris) distance was $0.48 \mathrm{~m}$, covering a scattering vector $(\mathrm{q}=4 \pi \sin (\theta) / \lambda)$ range from 0.008 to $0.6 \AA^{-1}$. A size exclusion buffer (50 mM potassium phosphate buffer $\mathrm{pH}$ 7.5) was used for the blank measurement. The dimeric form of the

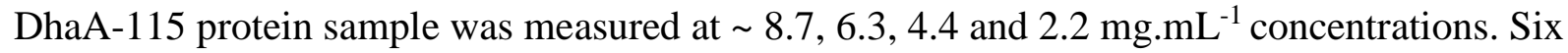
separate two-dimensional images were collected for buffer and sample ( 5 min exposure per image, 30 min total exposure). Radial averaging, data reduction and buffer subtractions were carried out using SAXSLab3.0.0r1, Rigaku. Six individual scattering curves (5 min exposures) were compared to check the radiation damage, then averaged. Integral structural parameters (supplemental Table S1) were determined using PRIMUS/qt ATSAS v.2.8.4 ${ }^{54}$. Data points before the Guinier region were truncated. Individual scattering curves from the concentration series were manually merged and truncated to a maximum of $q=0.4 \AA^{-1}$ for further analysis. Refined $a b$ initio models were produced by averaging the 10 individual $a b$ initio models produced by DAMMIF. The fixed core identified by DAMAVER was used as 
an input for DAMMIN modelling, where the computation mode was set to "slow" and all other parameters were kept at their defaults. Evaluation of solution scattering and fitting to experimental scattering curves was carried out using CRYSOL, where automatic constant subtraction was allowed; other parameters were kept at their defaults. Superimposition of the atomic and $a b$ initio models was carried out using SUPCOMB. Small-angle scattering datasets, experiment details, atomic model and fits have been saved to the Small Angle Scattering Biological Data Bank (www.sasbdb.org) ${ }^{55}$ as entry SASDHQ7.

Crosslinking Experiments. The SEC-separated monomeric and dimeric fractions of the DhaA115 enzyme were crosslinked separately in $50 \mathrm{mM}$ potassium phosphate buffer ( $\mathrm{pH} 7.5$ ) using the MS cleavable crosslinker, DSBU (CF Plus Chemicals, Czech Republic). The crosslinking reaction was carried out at $4{ }^{\circ} \mathrm{C}$ for one hour using a 1:100 protein:crosslinker ratio. Following crosslinking, complexes were either taken directly for in-solution trypsin digestion (sequencing grade, Promega) or subjected to SDS-PAGE (10\% gels) to separate crosslinked complexes. SDS-PAGE gels were stained with Biosafe Coomassie (Biorad, USA). Respective bands with crosslinked proteins were excised and further processed for overnight trypsin digestion. Resulting peptides and dipeptides were extracted into LC-MS vials using $2.5 \%$ formic acid (FA) in $50 \%$ acetonitrile (ACN) and $100 \% \mathrm{ACN}$ with the addition of polyethylene glycol $(0.001 \%)^{56}$, then concentrated in a SpeedVac concentrator (Thermo Fisher Scientific).

LC-MS/MS Analysis. LC-MS/MS analyses of all peptide mixtures were carried out using the RSLCnano system connected to an Orbitrap Fusion Lumos Tribrid mass spectrometer (Thermo Fisher Scientific). Before LC separation, tryptic digests were concentrated online and desalted using a trapping column $(100 \mu \mathrm{m} \times 30 \mathrm{~mm}$, column compartment temperature of $40{ }^{\circ} \mathrm{C}$ ) filled with $3.5 \mu \mathrm{m}$ X-Bridge BEH $130 \mathrm{C} 18$ sorbent (Waters). After washing of the trapping column with $0.1 \% \mathrm{FA}$, the peptides were eluted (flow $300 \mathrm{~nL}^{-\mathrm{min}^{-1}}$ ) from the trapping column into an analytical column (Acclaim Pepmap100 C18, $3 \mu \mathrm{m}$ particles, $75 \mu \mathrm{m}$ $\times 500 \mathrm{~mm}$; Thermo Fisher Scientific, column compartment temperature of $40{ }^{\circ} \mathrm{C}$ ) using a 50 min nonlinear gradient program (1-56\% of mobile phase B; mobile phase A: $0.1 \%$ FA in water; mobile phase B: $0.1 \% \mathrm{FA}$ in $80 \% \mathrm{ACN}$ ). Equilibration of the trapping column and the analytical column was completed before sample injection into the sample loop. The analytical column outlet was connected online to the Digital PicoView 550 (New Objective) ion source 
with sheath gas option and SilicaTip emitter active (New Objective; FS360-20-15-N-20-C12). ABIRD (Active Background Ion Reduction Device, ESI Source Solutions) was installed. MS data were acquired using a data-dependent strategy with cycle time of 3 seconds and with a survey scan $(300-1600 \mathrm{~m} / \mathrm{z})$. The resolution of the survey scan was $120000(200 \mathrm{~m} / \mathrm{z}) \mathrm{with}$ a maximum injection time of $50 \mathrm{~ms}$. Stepped HCD collision energies of 21, 27 and 33 were used for fragmentation of all precursors. MS/MS were recorded at 30,000 resolution with a maximum injection time of $150 \mathrm{~ms}$, and an isolation width of $1.4 \mathrm{~m} / \mathrm{z}^{57}$ Singly and doubly charged ions were excluded from fragmentation as crosslinked peptides are usually +3 or above. $^{58}$

MS Data Analysis. Raw files were used to create MGF files using the Proteome Discoverer software (Thermo Fisher Scientific; version 1.4) with in-house Mascot (Matrixscience, version 2.6). During MGF file generation, raw data were filtered out for cRAP proteins (based on http://www.thegpm.org/crap/; 112 sequences in total) to exclude common protein contaminants. MGF files were further processed in MeroX software for the identification of crosslinked peptides. For MeroX (Version: 2.0.1.3) searches, the following settings were used: crosslinker fragments: BuUr (+111.032028 Da) and Bu (+85.05276381 Da); specificity for site 1: $\mathrm{K}$ and $\mathrm{N}$-terminus; specificity for site 2: K,S,T,Y and N-terminus. Additionally, the RISE Mode was activated to compensate for 1 missing reporter doublet ion; MS1 accuracy: 10 ppm; MS2 accuracy: 25 ppm; enzyme used: trypsin; max. missed cleavages: arginine 3, Lysine 3; minimum peptide length: 5; max. modifications: 2; peptide mass: 200-6000 Da; static modifications: carbamidomethylation (cysteine, +57.021 Da); dynamic modifications: oxidation (methionine), deamidation (asparagine and glutamine). The false discovery rate (FDR) was set to $5 \%$. Decoy analysis was carried out by shuffling the FASTA database while keeping the amino acids of protease sites in place.

Thermal Unfolding and Refolding. DhaA115 monomer and dimer fractions were separated with SEC using a HiLoad S200 16/60 column (Cytiva); their purity was verified using native PAGE. For DSC experiments, samples were further dialyzed against $50 \mathrm{mM}$ potassium phosphate buffer $\mathrm{pH} 7.5$ overnight. All unfolding and refolding experiments were carried out using this buffer. Concentrations of the samples were 0.2 and $1 \mathrm{mg} \cdot \mathrm{mL}^{-1}$ for spectroscopic and DSC measurements, respectively. Unfolding transitions were found to be concentrationindependent in the 0.1-2 mg. $\mathrm{mL}^{-1}$ range tested. Temperature scanning experiments were 
carried out at a constant scan rate of $1^{\circ} \mathrm{C} \cdot \mathrm{min}^{-1}$ from 20 to $85{ }^{\circ} \mathrm{C}$, or with higher temperatures when possible. Heat capacity, ellipticity at $224 \mathrm{~nm}$ and the ratio of fluorescence intensities at 350 and $330 \mathrm{~nm}$ were recorded using a VP-Capillary DSC (Malvern Panalytical), Chirascan V100 spectrometer (Applied Photophysics) and NT.48 Prometheus (NanoTemper Technologies), respectively. The buffer-buffer scan baseline was subtracted from the proteinbuffer heat capacity data prior to their concentration normalization. Kinetics of unfolding was monitored by recording changes in ellipticity at $224 \mathrm{~nm}$ at different constant temperatures. A sample at room temperature was diluted 10 times with a pre-heated buffer to a final concentration of $0.2 \mathrm{mg} . \mathrm{mL}^{-1}$ and immediately transferred to the pre-heated cuvette placed in the instrument measurement chamber. The whole process took approx. $5 \mathrm{~s}$ which can be considered as the dead time of the measurement. The temperature of the solution was determined using a thermocouple inserted into the cuvette. Analysis of the oligomeric state of the samples after the kinetics monitoring was carried out with native PAGE. Refolding was carried out in several ways. First, samples $\left(0.2\right.$ and $\left.1 \mathrm{mg} \cdot \mathrm{mL}^{-1}\right)$ were heated and cooled down to different temperatures $\left(60-79^{\circ} \mathrm{C}\right)$ at $1{ }^{\circ} \mathrm{C} / \mathrm{min}$ scan rate. Their oligomeric state and activity were determined by native PAGE and Iwasaki assay, respectively. Next, dimer $\left(1 \mathrm{mg} \cdot \mathrm{mL}^{-1}\right)$ was kept at $62{ }^{\circ} \mathrm{C}$ in the Eppendorf tube and two aliquots were withdrawn at different times $(10,20,40,60,120$ and $240 \mathrm{~min})$. One aliquot was transferred to a chilled tube on ice whilst the second was allowed to equilibrate to room temperature on a benchtop. The oligomeric state of each aliquot was determined using native PAGE. Finally, the same experiment was repeated but with different concentrations of the dimer $\left(1,2,3,4,5,6\right.$ and $\left.6.6 \mathrm{mg} . \mathrm{mL}^{-1}\right)$ and 10 min incubation time.

Unfolding Data Analysis. Data from DSC, CD, fluorescence temperature scanning experiments and unfolding kinetics of the DhaA115 dimeric fraction were fitted globally to the four-state partially reversible model using MatLab version of the CalFitter ${ }^{30}$ software. The model involves the irreversible transition of the domain-swapped dimers to monomer subunits, followed by their reversible transition to the intermediate with subsequent irreversible denaturation. The dissection of the total $\Delta \mathrm{Cp}$ for each step was not feasible based on the data. Therefore, several dissections of $\Delta \mathrm{Cp}$ were made during fitting, for example, fixing it for one or more steps at 0 or at different values. This resulted in changes in the slope and shape of the $\Delta G^{\ddagger}(T)$ curve, especially at low temperatures. However, the estimation of energy barriers between 50 and $90^{\circ} \mathrm{C}$ where the transitions occur is very robust and relatively 
insensitive to the changes and dissection of $\Delta \mathrm{Cp}$. Deconvolution of the first DSC peak was achieved using the unfolding model with two parallel pathways included in the online version of CalFitter ( $->$ I1 -> D, N -> I2 -> D) $)^{30}$. The parameters for energy barriers of the first steps were set to zero and fixed, simulating the situation where two different species in solution irreversibly change to the same final state. The data were cut at $65^{\circ} \mathrm{C}$ and other parameters were allowed to vary. This model is perfectly valid since the dimers in solution are in an approximate 50/50 ratio. Data analysis of the DhaA115 monomer was carried out in the CalFitter using the three-state partially reversible model (Lumry-Eyring) ${ }^{30}$.

Chemical Denaturation Experiments. Denaturing buffer was prepared by dissolving urea in $50 \mathrm{mM} \mathrm{PB}$ pH 7.5 to a final concentration of $9 \mathrm{M}$. The precise concentration was determined using refractometry ${ }^{59}$. Next, a dilution series of urea was prepared between 0 and $9 \mathrm{M}$. DhaA115 monomer and dimer were added to each aliquot $\left(\mathrm{c}_{\text {final }}=0.2 \mathrm{mg} \cdot \mathrm{mL}^{-1}\right)$ and were allowed to equilibrate at $25{ }^{\circ} \mathrm{C}$ (approx. $24 \mathrm{~h}$ ). The fluorescence spectrum of each sample was collected between 300 and $400 \mathrm{~nm}$ after excitation at $266 \mathrm{~nm}$ using an UNcle instrument (Unchained labs). CD spectra were collected in the range $210-260 \mathrm{~nm}$ as an average of 5 measurements with $1 \mathrm{~nm}$ bandwidth and $0.5 \mathrm{~s}$ integration time. Denaturation curves were plotted as the average emission wavelength of the fluorescence spectra and ellipticity at 224 $\mathrm{nm}$ versus urea concentration. Data were fitted to the three-state reversible model using CDpal software ${ }^{60}$. For the dimer/monomer transition, the samples were equilibrated in $0-3 \mathrm{M}$ urea and, after equilibration for $24 \mathrm{~h}$, mixed in a 3:1 ratio with loading dye and analyzed for their oligomeric state using native PAGE. The fraction of monomer in each aliquot was calculated by densitometric analysis of the bands. Refolding was carried out in several ways. First, the monomer was partially or fully denatured in 5 or $9 \mathrm{M}$ urea, respectively, at various initial protein concentrations and subsequently diluted 10 times to native conditions. The oligomeric state was determined using native PAGE. Next, the kinetics of monomer refolding on dilution from denaturing to native conditions was probed by measuring changes in ellipticity at $224 \mathrm{~nm}$ over time. Finally, partially or fully denatured monomer was dialyzed to native conditions for $24 \mathrm{~h}$ and then analysis of the oligomeric state was carried out using native PAGE. Both unfolding and refolding were also repeated under reductive conditions by adding $1 \mathrm{mM}$ TCEP to the samples. 
Molecular Dynamics (MD) Simulations. The crystallographic structures of the DhaA115 dimers were used. The solvent and crystallization molecules were removed, and the double side chains were corrected to keep only the most populated conformation using the pdb4amber module of AmberTools $14^{61}$. The hydrogen atoms were added using the $\mathrm{H}++$ server $^{62}$, calculated in the implicit solvent at $\mathrm{pH} 7.5,0.1 \mathrm{M}$ salinity, an internal dielectric constant of 10 and external of 80 . The original crystallization solvent was added and the tLEaP program of AmberTools 14 was used to prepare the topology and coordinates files. For that, the force field ff $14 \mathrm{SB}^{63}$ was defined, $\mathrm{Na}^{+}$and $\mathrm{Cl}^{-}$ions were added to neutralize the system and achieve $0.10 \mathrm{M}$ concentration of $\mathrm{NaCl}$ salt, and an octagonal box of TIP3P ${ }^{64}$ water molecules with the edges at least $10 \AA$ away from the protein atoms was added. MD simulations were carried out using the PMEMD.CUDA ${ }^{65,66}$ module of AMBER $14^{61}$. In total, five minimization steps and twelve steps of equilibration dynamics were carried out prior to the production MD. The first four minimization steps, composed of 2,500 cycles of steepest descent followed by 7,500 cycles of the conjugate gradient, were carried out as follows: (i) in the first step, all the atoms of the protein and ligand were restrained with $500 \mathrm{kcal} . \mathrm{mol}^{-1} . \AA^{2}$ harmonic force constant; (ii) in the remaining steps, only the backbone atoms of the protein and heavy atoms of the ligand were restrained, respectively, with 500, 125, and $25 \mathrm{kcal}^{\mathrm{mol}}{ }^{-}$ ${ }^{1} . \AA^{2}$ force constant. A fifth minimization step, composed of 5,000 cycles of steepest descent and 15,000 cycles of the conjugate gradient, was carried out without any restraints. The subsequent MD simulations used periodic boundary conditions, the particle mesh Ewald method for treatment of the long-range interactions beyond the $10 \AA$ cutoff $^{67}$, the SHAKE algorithm $^{68}$ to constrain the bonds involving the hydrogen atoms, a Langevin thermostat with collision frequency $1.0 \mathrm{ps}^{-1}$, and a time step of $2 \mathrm{fs}$. Equilibration dynamics were carried out in twelve steps: (i) 20 ps of gradual heating from 0 to $310 \mathrm{~K}$, under constant volume, restraining the protein atoms and ligand with $200 \mathrm{kcal} \cdot \mathrm{mol}^{-1} . \AA^{2}$ harmonic force constant; (ii) ten MDs of 400 ps each, at constant pressure (1 bar) and constant temperature $(310 \mathrm{~K})$, with gradually decreasing restraints on the backbone atoms of the protein and heavy atoms of the ligand with harmonic force constants of $150,100,75,50,25,15,10,5,1$, and $0.5 \mathrm{kcal}_{\mathrm{mol}}{ }^{-1} . \AA^{2}$; (iii) 400 ps of unrestrained MD at the same conditions as the previous restrained MDs. The energy and coordinates were saved every $10 \mathrm{ps}$. The production MDs were run for $100 \mathrm{~ns}$ using the same settings employed in the last equilibration step and carried out in duplicate for each system. The trajectories were analyzed using the cpptraj $^{69}$ module of AmberTools 14 and visualized using PyMOL 1.7.4 $4^{47}$ and VMD 1.9.1 ${ }^{70}$. The simulations of each type were combined in a 
single one using cpptraj $^{69}$, aligned to the respective crystal structures by minimizing the rootmean-square deviation (RMSD) of the backbone atoms $\left(C, N, O, C_{\alpha}\right)$, excluding the very flexible terminal residues of each chain (5-7 terminal residues). The B-factors were calculated for the backbone atoms of each system, by stepwise fitting each catalytic unit as follows: i) for the DhaA115 monomer, calculated for residues 9-289 after aligning the simulation by fitting the same atoms; ii) for DhaA115-DSD1, calculated for residues A:10-141 + B:133290 (the entire catalytic unit from site $\mathrm{A}+$ hinge) by aligning A:10-133 + B:143-290 (catalytic unit from site A), and for residues A:142-290 + B:10-132 (catalytic unit from site B) by aligning the same residues; iii) for DhaA115-DSD2, calculated for residues A:10-199+ B:133-290 (catalytic unit from site A + hinge) by aligning A:10-192 + B:200-289 (catalytic unit from site A), and for residues A:200-290 + B:10-192 (catalytic unit from site B) by aligning the same residues. This was done in order to exclude the fluctuations due to the tilting and rocking movements of the units with respect to each other, and thus obtain normalized B-factors more comparable to the monomeric unit. The MD trajectories of the dimers were clustered using cpptraj $^{69}$, with distance-based metrics of the mass-weighted RMSD for all the heavy atoms excluding the highly flexible terminal residues (residues 9-289 were used). The hierarchical agglomerative clustering algorithm was used with average linkage, epsilon 1.5 and sieve 5, and a minimum of 3 clusters. The centroid structures of the different clusters were used for visualization.

\section{DATA AND CODE AVAILABILITY}

Atomic coordinates and structural factors have been saved in the Protein Data Bank (www.wwpdb.org) ${ }^{49}$ under PDB accession codes: 6TY7, 6XT8 and 6XTC. SAXS datasets, experiment details, atomic model and fits have been saved in the Small Angle Scattering Biological Data Bank (www.sasbdb.org) ${ }^{55}$ as entry SASDHQ7. Authors will release the atomic coordinates and experimental data upon article publication.

\section{AUTHOR CONTRIBUTIONS}

K. M. and K. C. prepared the protein samples for crystallization, carried out the initial crystallization screenings, and optimized crystallization hits. K. M. prepared the protein samples for SAXS. K. M., K. C., R. C. and M. M. collected diffraction data and solved the 
protein crystal structures. A. K. and M. H. carried out thermal and chemical denaturation experiments. M. V. measured temperature profiles. S. M. M. and D. B. carried out MD analyses. P. B., K. C. and R.C. carried out enzyme kinetics experiments. J. I. P. helped with the literature searches. M. M., Z. P. and J. D. designed the project, supervised research and interpreted data. K. M., A. K. and M. M. wrote the manuscript with contributions from all the authors. All authors have approved the final version of the manuscript.

\section{CONFLICT OF INTEREST}

The authors declare no competing financial interest.

\section{ACKNOWLEDGEMENTS}

The authors would like to express their thanks to the Czech Ministry of Education (CZ.02.1.01/0.0/0.0/17_043/0009632， CZ.02.1.01/0.0/0.0/16_026/0008451， LM2018121, LM2018131). This project has received funding from the European Union's Horizon 2020 research and innovation program (Nos. 857560 and 814418). This project has received funding from the European Union's Horizon 2020 research and innovation program under the Marie Sklodowska-Curie grant agreement No. 792772. M. M. acknowledges financial support from GAMU of the Masaryk University (MUNI/H/1561/2018). The computational resources were supplied by the project “e-Infrastruktura CZ" (e-INFRA LM2018140) provided within the program Projects of Large Research, Development and Innovations Infrastructures. CIISB research infrastructure project (LM2018127) is acknowledged for financial support of the measurements at the X-ray Diffraction and Bio-SAXS Core Facility, Biomolecular Interactions and Crystallization Core Facility. We thank Tomas Klumpler (CEITEC-MU, Brno, Czech Republic) for his assistance during SAXS data collection and processing. The crystallographic experiments were carried out using beamline ID23-1 at the European Synchrotron Facility (ESRF) in Grenoble (France), using beamline I03 at the Diamond Light Source in Didcot (United Kingdom), and using the PXIII beamline at the Swiss Light Source (SLS) in Villigen (Switzerland). We are grateful to the members of the synchrotron facilities for the use of their beamlines and help during data collection. 


\section{REFERENCES}

(1) Kazlauskas, R. Engineering More Stable Proteins. Chem. Soc. Rev. 2018, 47 (24), 9026-9045.

(2) Bommarius, A. S.; Paye, M. F. Stabilizing Biocatalysts. Chem. Soc. Rev. 2013, 42 (15), 6534.

(3) Goldenzweig, A.; Fleishman, S. J. Principles of Protein Stability and Their Application in Computational Design. Annu. Rev. Biochem. 2018, 87 (1), 105-129.

(4) Musil, M.; Konegger, H.; Hon, J.; Bednar, D.; Damborsky, J. Computational Design of Stable and Soluble Biocatalysts. ACS Catal. 2019, 9 (2), 1033-1054.

(5) Bednar, D.; Beerens, K.; Sebestova, E.; Bendl, J.; Khare, S.; Chaloupkova, R.; Prokop, Z.; Brezovsky, J.; Baker, D.; Damborsky, J. FireProt: Energy- and Evolution-Based Computational Design of Thermostable Multiple-Point Mutants. PLoS Comput. Biol. 2015, 11 (11), e1004556.

(6) Wijma, H. J.; Floor, R. J.; Jekel, P. A.; Baker, D.; Marrink, S. J.; Janssen, D. B. Computationally Designed Libraries for Rapid Enzyme Stabilization. Protein Eng. Des. Sel. 2014, 27 (2), 49-58.

(7) Bennett, M. J.; Choe, S.; Eisenberg, D. Domain Swapping: Entangling Alliances between Proteins. Proc. Natl. Acad. Sci. U.S.A. 1994, 91 (8), 3127-3131.

(8) Bennett, M. J.; Schlunegger, M. P.; Eisenberg, D. 3D Domain Swapping: A Mechanism for Oligomer Assembly. Protein Sci. 1995, 4 (12), 2455-2468.

(9) Gronenborn, A. M. Protein Acrobatics in Pairs - Dimerization via Domain Swapping. Curr. Opin. Struc. Biol. 2009, 19 (1), 39-49.

(10) Rousseau, F.; Schymkowitz, J. W. H.; Itzhaki, L. S. The Unfolding Story of Three-Dimensional Domain Swapping. Structure 2003, 11 (3), 243-251.

(11) Liu, L.; Byeon, I.-J. L.; Bahar, I.; Gronenborn, A. M. Domain Swapping Proceeds via Complete Unfolding: A ${ }^{19} \mathrm{~F}$ - and ${ }^{1}$ H-NMR Study of the Cyanovirin-N Protein. J. Am. Chem. Soc. 2012, 134 (9), 4229-4235.

(12) Liu, Z.; Huang, Y. Evidences for the Unfolding Mechanism of Three-Dimensional Domain Swapping: The Unfolding Mechanism of 3D Domain Swapping. Protein Sci. 2013, 22 (3), 280-286.

(13) Mascarenhas, N. M.; Gosavi, S. Understanding Protein Domain-Swapping Using StructureBased Models of Protein Folding. Prog. Biophys. Mol. Biol. 2017, 128, 113-120.

(14) Liu, Y.; Eisenberg, D. 3D Domain Swapping: As Domains Continue to Swap. Protein Sci. 2002, 11 (6), 1285-1299.

(15) Shiga, S.; Yamanaka, M.; Fujiwara, W.; Hirota, S.; Goda, S.; Makabe, K. Domain-Swapping Design by Polyproline Rod Insertion. ChemBioChem 2019, 20 (19), 2454-2457. 
(16) Nandwani, N.; Surana, P.; Negi, H.; Mascarenhas, N. M.; Udgaonkar, J. B.; Das, R.; Gosavi, S. A Five-Residue Motif for the Design of Domain Swapping in Proteins. Nat. Commun. 2019, 10 (1).

(17) Mizuno, H.; Fujimoto, Z.; Koizumi, M.; Kano, H.; Atoda, H.; Morita, T. Structure of Coagulation Factors IX/X-Binding Protein, a Heterodimer of C-Type Lectin Domains. Nat. Struct. Biol. 1997, 4 (6), 438-441.

(18) Chen, L.-Y.; Huang, Y.-C.; Huang, S.-T.; Hsieh, Y.-C.; Guan, H.-H.; Chen, N.-C.; Chuankhayan, P.; Yoshimura, M.; Tai, M.-H.; Chen, C.-J. Domain Swapping and SMYD1 Interactions with the PWWP Domain of Human Hepatoma-Derived Growth Factor. Sci. Rep. 2018, 8 (1).

(19) Park, C. K.; Joshi, H. K.; Agrawal, A.; Ghare, M. I.; Little, E. J.; Dunten, P. W.; Bitinaite, J.; Horton, N. C. Domain Swapping in Allosteric Modulation of DNA Specificity. PLoS Biol. 2010, 8 (12), e1000554.

(20) Zhou, X.; Wang, H.; Zhang, Y.; Gao, L.; Feng, Y. Alteration of Substrate Specificities of Thermophilic $\alpha / \beta$ Hydrolases through Domain Swapping and Domain Interface Optimization. Acta Biochim. Biophys. Sin. 2012, 44 (12), 965-973.

(21) Karchin, J. M.; Ha, J.-H.; Namitz, K. E.; Cosgrove, M. S.; Loh, S. N. Small Molecule-Induced Domain Swapping as a Mechanism for Controlling Protein Function and Assembly. Sci. Rep. 2017, 7 (1).

(22) Nussinov, R.; Xu, D.; Tsai, C.-J. Mechanism and Evolution of Protein Dimerization: Protein Dimerization. Protein Sci. 1998, 7 (3), 533-544.

(23) Bennett, M. J.; Sawaya, M. R.; Eisenberg, D. Deposition Diseases and 3D Domain Swapping. Structure 2006, 14 (5), 811-824.

(24) Janowski, R.; Kozak, M.; Abrahamson, M.; Grubb, A.; Jaskolski, M. 3D Domain-Swapped Human Cystatin C with Amyloidlike Intermolecular $\beta$-Sheets. Proteins 2005, 61 (3), 570-578.

(25) Knaus, K. J.; Morillas, M.; Swietnicki, W.; Malone, M.; Surewicz, W. K.; Yee, V. C. Crystal Structure of the Human Prion Protein Reveals a Mechanism for Oligomerization. Nat. Struct Biol. 2001, 8 (9), 770-774.

(26) Markova, K.; Chmelova, K.; Marques, S. M.; Carpentier, P.; Bednar, D.; Damborsky, J.; Marek, M. Decoding the Intricate Network of Molecular Interactions of a Hyperstable Engineered Biocatalyst. Chem. Sci. 2020, 11 (41), 11162-11178.

(27) Beerens, K.; Mazurenko, S.; Kunka, A.; Marques, S. M.; Hansen, N.; Musil, M.; Chaloupkova, R.; Waterman, J.; Brezovsky, J.; Bednar, D.; Prokop, Z.; Damborsky, J. Evolutionary Analysis as a Powerful Complement to Energy Calculations for Protein Stabilization. ACS Catal. 2018, 8 (10), 9420-9428. 
(28) Sanchez-Ruiz, J. M. Theoretical Analysis of Lumry-Eyring Models in Differential Scanning Calorimetry. Biophys. J. 1992, 61 (4), 921-935.

(29) Mazurenko, S.; Kunka, A.; Beerens, K.; Johnson, C. M.; Damborsky, J.; Prokop, Z. Exploration of Protein Unfolding by Modelling Calorimetry Data from Reheating. Sci. Rep. 2017, 7 (1).

(30) Mazurenko, S.; Stourac, J.; Kunka, A.; Nedeljković, S.; Bednar, D.; Prokop, Z.; Damborsky, J. CalFitter: A Web Server for Analysis of Protein Thermal Denaturation Data. Nucleic Acids Res. 2018, 46 (W1), W344-W349.

(31) Chaloupkova, R.; Sykorova, J.; Prokop, Z.; Jesenska, A.; Monincova, M.; Pavlova, M.; Tsuda, M.; Nagata, Y.; Damborsky, J. Modification of Activity and Specificity of Haloalkane Dehalogenase from Sphingomonas Paucimobilis UT26 by Engineering of Its Entrance Tunnel. J. Biol. Chem. 2003, 278 (52), 52622-52628.

(32) Pavlova, M.; Klvana, M.; Prokop, Z.; Chaloupkova, R.; Banas, P.; Otyepka, M.; Wade, R. C.; Tsuda, M.; Nagata, Y.; Damborsky, J. Redesigning Dehalogenase Access Tunnels as a Strategy for Degrading an Anthropogenic Substrate. Nat. Chem. Biol. 2009, 5 (10), 727-733.

(33) Musil, M.; Stourac, J.; Bendl, J.; Brezovsky, J.; Prokop, Z.; Zendulka, J.; Martinek, T.; Bednar, D.; Damborsky, J. FireProt: Web Server for Automated Design of Thermostable Proteins. Nucleic Acids Res. 2017, 45 (1), W393-W399.

(34) Zegers, I.; Deswarte, J.; Wyns, L. Trimeric Domain-Swapped Barnase. Proc. Natl. Acad. Sci. U.S.A. 1999, 96 (3), 818-822.

(35) Bergdoll, M.; Remy, M.-H.; Cagnon, C.; Masson, J.-M.; Dumas, P. Proline-Dependent Oligomerization with Arm Exchange. Structure 1997, 5 (3), 391-401.

(36) Bennett, M. J.; Eisenberg, D. The Evolving Role of 3D Domain Swapping in Proteins. Structure 2004, 12 (8), 1339-1341.

(37) Jaskólski, M. 3D Domain Swapping, Protein Oligomerization, and Amyloid Formation. Acta Biochim. Pol. 2001, 48 (4), 807-827.

(38) Nurizzo, D.; Mairs, T.; Guijarro, M.; Rey, V.; Meyer, J.; Fajardo, P.; Chavanne, J.; Biasci, J.C.; McSweeney, S.; Mitchell, E. The ID23-1 Structural Biology Beamline at the ESRF. $J$. Synchrotron. Rad. 2006, 13 (3), 227-238.

(39) Kabsch, W. XDS. Acta Crystallogr. D Biol. Crystallogr. 2010, 66 (2), 125-132.

(40) Evans, P. R.; Murshudov, G. N. How Good Are My Data and What Is the Resolution? Acta Crystallogr. D Biol. Crystallogr. 2013, 69 (7), 1204-1214.

(41) McCoy, A. J.; Grosse-Kunstleve, R. W.; Adams, P. D.; Winn, M. D.; Storoni, L. C.; Read, R. J. Phaser Crystallographic Software. J. Appl. Crystallogr. 2007, 40 (4), 658-674.

(42) Adams, P. D.; Afonine, P. V.; Bunkóczi, G.; Chen, V. B.; Davis, I. W.; Echols, N.; Headd, J. J.; Hung, L.-W.; Kapral, G. J.; Grosse-Kunstleve, R. W.; McCoy, A. J.; Moriarty, N. W.; Oeffner, 
R.; Read, R. J.; Richardson, D. C.; Richardson, J. S.; Terwilliger, T. C.; Zwart, P. H. PHENIX:

A Comprehensive Python-Based System for Macromolecular Structure Solution. Acta Crystallogr. D Biol. Crystallogr. 2010, 66 (2), 213-221.

(43) Lahoda, M.; Mesters, J. R.; Stsiapanava, A.; Chaloupkova, R.; Kuty, M.; Damborsky, J.; Kuta Smatanova, I. Crystallographic Analysis of 1,2,3-Trichloropropane Biodegradation by the Haloalkane Dehalogenase DhaA31. Acta Crystallogr. D Biol. Crystallogr. 2014, 70 (2), 209217.

(44) Afonine, P. V.; Grosse-Kunstleve, R. W.; Echols, N.; Headd, J. J.; Moriarty, N. W.; Mustyakimov, M.; Terwilliger, T. C.; Urzhumtsev, A.; Zwart, P. H.; Adams, P. D. Towards Automated Crystallographic Structure Refinement with Phenix.Refine. Acta Crystallogr. D: Biol. Crystallogr. 2012, 68 (4), 352-367.

(45) Emsley, P.; Cowtan, K. Coot: Model-Building Tools for Molecular Graphics. Acta Crystallogr. D Biol. Crystallogr. 2004, 60 (12), 2126-2132.

(46) Williams, C. J.; Headd, J. J.; Moriarty, N. W.; Prisant, M. G.; Videau, L. L.; Deis, L. N.; Verma, V.; Keedy, D. A.; Hintze, B. J.; Chen, V. B.; Jain, S.; Lewis, S. M.; Arendall, W. B.; Snoeyink, J.; Adams, P. D.; Lovell, S. C.; Richardson, J. S.; Richardson, D. C. MolProbity: More and Better Reference Data for Improved All-Atom Structure Validation. Protein Sci. 2018, 27 (1), 293-315.

(47) Schrödinger, LLC. The PyMOL Molecular Graphics System.

(48) Krissinel, E.; Henrick, K. Secondary-Structure Matching (SSM), a New Tool for Fast Protein Structure Alignment in Three Dimensions. Acta Crystallogr. D Biol. Crystallogr. 2004, 60 (12), 2256-2268.

(49) wwPDB consortium; Burley, S. K.; Berman, H. M.; Bhikadiya, C.; Bi, C.; Chen, L.; Costanzo, L. D.; Christie, C.; Duarte, J. M.; Dutta, S.; Feng, Z.; Ghosh, S.; Goodsell, D. S.; Green, R. K.; Guranovic, V.; Guzenko, D.; Hudson, B. P.; Liang, Y.; Lowe, R.; Peisach, E.; Periskova, I.; Randle, C.; Rose, A.; Sekharan, M.; Shao, C.; Tao, Y.-P.; Valasatava, Y.; Voigt, M.; Westbrook, J.; Young, J.; Zardecki, C.; Zhuravleva, M.; Kurisu, G.; Nakamura, H.; Kengaku, Y.; Cho, H.; Sato, J.; Kim, J. Y.; Ikegawa, Y.; Nakagawa, A.; Yamashita, R.; Kudou, T.; Bekker, G.-J.; Suzuki, H.; Iwata, T.; Yokochi, M.; Kobayashi, N.; Fujiwara, T.; Velankar, S.; Kleywegt, G. J.; Anyango, S.; Armstrong, D. R.; Berrisford, J. M.; Conroy, M. J.; Dana, J. M.; Deshpande, M.; Gane, P.; Gáborová, R.; Gupta, D.; Gutmanas, A.; Koča, J.; Mak, L.; Mir, S.; Mukhopadhyay, A.; Nadzirin, N.; Nair, S.; Patwardhan, A.; Paysan-Lafosse, T.; Pravda, L.; Salih, O.; Sehnal, D.; Varadi, M.; Vařeková, R.; Markley, J. L.; Hoch, J. C.; Romero, P. R.; Baskaran, K.; Maziuk, D.; Ulrich, E. L.; Wedell, J. R.; Yao, H.; Livny, M.; Ioannidis, Y. E. Protein Data Bank: The Single Global Archive for 3D Macromolecular Structure Data. Nucleic Acids Research 2019, 47 (D1), D520-D528. 
(50) Schuck, P. Size-Distribution Analysis of Macromolecules by Sedimentation Velocity Ultracentrifugation and Lamm Equation Modeling. Biophys. J. 2000, 78 (3), 1606-1619.

(51) Brautigam, C. A. Calculations and Publication-Quality Illustrations for Analytical Ultracentrifugation Data. In Methods in Enzymology; Elsevier, 2015; Vol. 562, pp 109-133.

(52) Iwasaki, I.; Utsumi, S.; Ozawa, T. New Colorimetric Determination of Chloride Using Mercuric Thiocyanate and Ferric Ion. Bull. Chem. Soc. Jpn 1952, 25 (3), 226-226.

(53) Buryska, T.; Vasina, M.; Gielen, F.; Vanacek, P.; van Vliet, L.; Jezek, J.; Pilat, Z.; Zemanek, P.; Damborsky, J.; Hollfelder, F.; Prokop, Z. Controlled Oil/Water Partitioning of Hydrophobic Substrates Extending the Bioanalytical Applications of Droplet-Based Microfluidics. Anal. Chem. 2019, 91 (15), 10008-10015.

(54) Franke, D.; Petoukhov, M. V.; Konarev, P. V.; Panjkovich, A.; Tuukkanen, A.; Mertens, H. D. T.; Kikhney, A. G.; Hajizadeh, N. R.; Franklin, J. M.; Jeffries, C. M.; Svergun, D. I. ATSAS 2.8: A Comprehensive Data Analysis Suite for Small-Angle Scattering from Macromolecular Solutions. J. Appl. Crystallogr. 2017, 50 (4), 1212-1225.

(55) Valentini, E.; Kikhney, A. G.; Previtali, G.; Jeffries, C. M.; Svergun, D. I. SASBDB, a Repository for Biological Small-Angle Scattering Data. Nucleic Acids Res. 2015, 43 (D1), D357-D363.

(56) Stejskal, K.; Potesil, D.; Zdrahal, Z. Suppression of Peptide Sample Losses in Autosampler Vials. J. Proteome Res. 2013, 12 (6), 3057-3062.

(57) Stieger, C. E.; Doppler, P.; Mechtler, K. Optimized Fragmentation Improves the Identification of Peptides Cross-Linked by MS-Cleavable Reagents. J. Proteome Res. 2019, 18 (3), 13631370 .

(58) Giese, S. H.; Fischer, L.; Rappsilber, J. A Study into the Collision-Induced Dissociation (CID) Behavior of Cross-Linked Peptides. Mol. Cell. Proteomics 2016, 15 (3), 1094-1104.

(59) Pace, C. N. Determination and Analysis of Urea and Guanidine Hydrochloride Denaturation Curves. In Methods in Enzymology; Elsevier, 1986; Vol. 131, pp 266-280.

(60) Niklasson, M.; Andresen, C.; Helander, S.; Roth, M. G. L.; Zimdahl Kahlin, A.; Lindqvist Appell, M.; Mårtensson, L.-G.; Lundström, P. Robust and Convenient Analysis of Protein Thermal and Chemical Stability: Robust and Convenient Analysis of Protein Stability. Protein Sci. 2015, 24 (12), 2055-2062.

(61) Case, D. A.; Berryman, J. T.; Betz, R. M.; Cai, Q.; Cerutti, D. S.; Cheatham, T. E.; Darden, T. A.; Duke, R. E.; Gohlke, H.; Goetz, A. W.; Gusarov, S.; Homeyer, N.; Janowski, P.; Kaus, J.; Kolossváry, I.; Kovalenko, A.; Lee, T. S.; LeGrand, S.; Luchko, T.; Luo, R.; Madej, B.; Merz, K. M.; Paesani, F.; Roe, D. R.; Roitberg, A.; Sagui, C.; Salomon-Ferrer, R.; Seabra, G.; Simmerling, C. L.; Smith, W.; Swails, J.; Walker, R. C.; Wang, J.; Wolf, R. M.; Wu, X.; Kollman, P. A. AMBER 14, University of California, San Francisco. 2014. 
(62) Gordon, J. C.; Myers, J. B.; Folta, T.; Shoja, V.; Heath, L. S.; Onufriev, A. H++: A Server for Estimating PKas and Adding Missing Hydrogens to Macromolecules. Nucleic Acids Res. 2005, 33, W368-W371.

(63) Maier, J. A.; Martinez, C.; Kasavajhala, K.; Wickstrom, L.; Hauser, K. E.; Simmerling, C. Ff14SB: Improving the Accuracy of Protein Side Chain and Backbone Parameters from Ff99SB. J. Chem. Theory Comput. 2015, 11 (8), 3696-3713.

(64) Jorgensen, W. L.; Chandrasekhar, J.; Madura, J. D.; Impey, R. W.; Klein, M. L. Comparison of Simple Potential Functions for Simulating Liquid Water. J. Chem. Phys. 1983, 79 (2), 926-935.

(65) Götz, A. W.; Williamson, M. J.; Xu, D.; Poole, D.; Le Grand, S.; Walker, R. C. Routine Microsecond Molecular Dynamics Simulations with AMBER on GPUs. 1. Generalized Born. J. Chem. Theory Comput. 2012, 8 (5), 1542-1555.

(66) Le Grand, S.; Götz, A. W.; Walker, R. C. SPFP: Speed without Compromise-A Mixed Precision Model for GPU Accelerated Molecular Dynamics Simulations. Comput. Phys. Commun. 2013, 184 (2), 374-380.

(67) Darden, T.; York, D.; Pedersen, L. Particle Mesh Ewald: An N·log(N) Method for Ewald Sums in Large Systems. J. Chem. Phys. 1993, 98 (12), 10089-10092.

(68) Ryckaert, J.-P.; Ciccotti, G.; Berendsen, H. J. C. Numerical Integration of the Cartesian Equations of Motion of a System with Constraints: Molecular Dynamics of n-Alkanes. $J$. Comput. Phy. 1977, 23 (3), 327-341.

(69) Roe, D. R.; Cheatham, T. E. PTRAJ and CPPTRAJ: Software for Processing and Analysis of Molecular Dynamics Trajectory Data. J. Chem. Theory Comput. 2013, 9 (7), 3084-3095.

(70) Humphrey, W.; Dalke, A.; Schulten, K. VMD: Visual Molecular Dynamics. J. Mol. Graph. 1996, 14 (1), 33-38. 